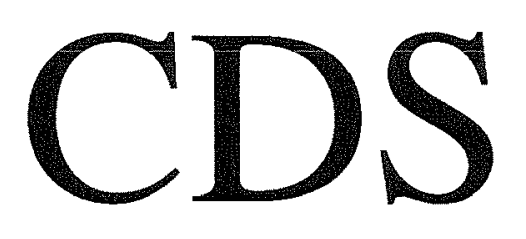

TECHNICAL MEMORANDUM NO. CIT-CDS 97-014

November, 1997

\title{
"Nonlinear Rescaling of Control Laws with Application to Stabilization in the Presence of Magnitude Saturation"
}

Pascal Morin and Richard M. Murray and L. Praly

Control and Dynamical Systems

California Institute of Technology

Pasadena, California 91125 


\title{
Nonlinear Rescaling of Control Laws with Application to Stabilization in the Presence of Magnitude Saturation
}

\author{
P. Morin* $\quad$ R.M. Murray ${ }^{\dagger} \quad$ L. Praly ${ }^{\ddagger}$ \\ Submitted, 4th IFAC Nonlinear Control Systems Design Symposium
}

\begin{abstract}
Motivated by some recent results on the stabilization of homogeneous systems, we present a gain-scheduling approach for the stabilization of non-linear systems. Given a one-parameter family of stabilizing feedbacks and associated Lyapunov functions, we show how the parameter can be rescaled as a function of the state to give a new stabilizing controller. In the case of homogeneous systems, we obtain generalizations of some existing results. We show that this approach can also be applied to nonhomogeneous systems. In particular, the main application considered in this paper is to the problem of stabilization with magnitude limitations. For this problem, we develop a design method for single-input controllable systems with eigenvalues in the left closed plane.
\end{abstract}

Keywords: gain scheduling, global stabilization, bounded control, nonlinear feedback.

\section{Introduction}

The problem of stabilization with control limitations is crucial in many applications while, even for otherwise linear systems, it cannot be solved with standard linear techniques. For controllable linear systems subject to magnitude limitations on the inputs, globally asymptotically stabilizing feedbacks exist if and only if the open loop system has no eigenvalues in the open right plane. Under this assumption, several design methods have recently been developed (see e.g. $[16,17,15,10,8]$ and the references therein). Although different in their approach and characteristics, all these methods rely on some kind of gain scheduling, i.e. the control can be viewed as a linear feedback with gains converging to zero as the norm of the state converges to infinity. The difficulty of the control design is twofold: on one hand, the stability of the system must be preserved while rescaling the gains, on the other hand the rescaled controller must satisfy the control limitations.

*INRIA, 06902 Sophia-Antipolis Cedex, FRANCE. E-mail: pmorin@sophia.inria.fr. This author contributed to this work while he was with the CDS Dept. at Caltech as a Post-doctoral fellow.

${ }^{\dagger}$ Control and Dynamical System (CDS), California Institute of Technology, Pasadena, CA 91125, USA. E-mail: murray@indra.caltech.edu.

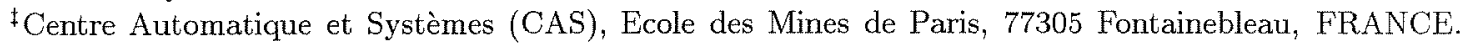
E-mail: praly@cas.ensmp.fr. 
Recently, in [9], M'Closkey and Murray have given a sufficient condition which ensures that a smooth feedback (stabilizing a driftless control system of homogeneous vector fields) can be rescaled into a homogeneous feedback yielding exponential stability (for driftless systems, this latter property cannot be obtained with smooth feedback when the number of states is larger than the number of control vector fields). A similar approach was also developed independently by Praly in [12] for more general forms of homogeneity. In this paper, we extend this approach to general systems, i.e. not necessarily homogeneous. For homogeneous systems, we obtain some generalization of [9, Th. 4], but the main application that we consider is to the problem of stabilization with magnitude limitations. For single-input linear controllable systems, we design bounded feedbacks which ensure global stabilization of the controlled system. In this case, the controller is just a one-parameter family of linear controllers, with the parameter properly scaled as a function of the state. This gives a rather simple controller and requires very little on-line computation: only the scaling parameter is not explicitly defined as a function of the state. Also, the knowledge of an explicit family of (non-increasing) Lyapunov functions gives us quantitative information about the controlled system. Our approach to this problem has several connections with other works. First, it can be compared with the approach of Megretski and Lin $[10,8]$ in the sense that we also use a monotonic family of Lyapunov functions. Because we only require these functions to be non-increasing along the trajectories of the controlled system, we can find an explicit family of Lyapunov functions and more explicit control laws. As a counterpart, taking into account the magnitude limitations is much harder than in $[10,8]$. Also, in the special case of a chain of integrators, our family of controllers is basically the same as that used by Lauvdal and al. [6]. This suggests a way to extend the approach of [6] to more general systems.

The paper is organized as follows. A simple motivating example is treated in Section 2. We present in Section 3 the main result on the rescaling of control law. In Section 4, we show how this result can be used for some stabilization problems of homogeneous systems. In particular, we recover and extend the results of [9, Th. 4]. The main application is considered in Section 5 where we develop a design method for the stabilization of singleinput linear systems with control limitations. An illustrative example is treated in Section 6, and some concluding remarks are given in Section 7. The proofs of the main result and of several technical lemmas are given in Appendix.

The following notation will be used.

$\operatorname{Id}(n)$ denotes the identity matrix in $\mathbb{R}^{n}$. For any matrix $M, M_{i}$ denotes the $i$-th row of $M$, and $M^{i}$ denotes the upper left minor of order $i$. For any vector $\left(d_{1}, \ldots, d_{n}\right)$ in $\mathbb{R}^{n}, \operatorname{Diag}\left(d_{i}\right)$ denotes the diagonal matrix with $d_{i}$ as $(i, i)$-th entry.

By convention, $\sum_{\emptyset}=0$ and $\prod_{\emptyset}=1$.

$\mathbb{R}_{+}$denotes the set of strictly positive real numbers.

For any set $A \in \mathbb{R}^{m}, A^{o}$ denotes the interior of $A$. 


\section{Motivating example}

Consider the following system in $\mathbb{R}^{2}$ :

$$
\left\{\begin{array}{l}
\dot{x}_{1}=x_{2} \\
\dot{x}_{2}=u
\end{array}\right.
$$

together with any linear stabilizing controller $u(x)=-a_{1} x_{1}-a_{2} x_{2} \quad\left(a_{1}, a_{2}>0\right)$, and suppose that we want to find a bounded globally asymptotically stabilizing feedback for (1). For any $\lambda>0$, the controller

$$
u(\lambda, x)=-\frac{a_{1}}{\lambda^{2}} x_{1}-\frac{a_{2}}{\lambda} x_{2}
$$

is a stabilizing feedback for (1), and the Lyapunov function

$$
V(\lambda, x)=\frac{a_{1}}{\lambda^{4}} x_{1}^{2}+\frac{1}{\lambda^{2}} x_{2}^{2}
$$

is non-increasing along the trajectories of the controlled system (1)-(2). We note that the rescaling of $u$ and $V$ is nonlinear in $\lambda$. Its particular form is due to the homogeneity properties of the system, as discussed in Section 4 . Consider the equation in $\lambda$

$$
V(\lambda, x)=1 \text {. }
$$

For any $x \neq 0$, this equation has a unique positive solution

$$
\lambda=\left[\frac{x_{2}^{2}+\left(x_{2}^{4}+4 a_{1} x_{1}^{2}\right)^{\frac{1}{2}}}{2}\right]^{\frac{1}{2}} .
$$

Consider now the feedback $u(\lambda(x), x)$ with $u(\lambda, x)$ defined by $(2)$ and $\lambda(x)$ defined by

$$
\lambda(x)= \begin{cases}1 & \text { if } V(1, x) \leq 1 \\ \text { the solution }(5) \text { of }(4) & \text { otherwise. }\end{cases}
$$

We claim that this feedback is bounded and ensures global asymptotic stability of (1). The boundedness is easily verified since, from (2) and (5), $\frac{x_{1}}{\lambda^{2}(x)}$ and $\frac{x_{2}}{\lambda(x)}$ are bounded. The asymptotic stability of the closed loop system relies on the following fact. Since $\frac{\partial V}{\partial \lambda}<0$ for any $x \neq 0$ and $\lambda>0$, and since for $V(1, x)>1, V(\lambda(x), x)=1$, we obtain by differentiating this last equality that:

$$
\frac{\partial \lambda}{\partial x} \dot{x}=-\left[\frac{\partial V}{\partial \lambda}\right]^{-1} \frac{\partial V}{\partial x} \dot{x} \leq 0
$$

This implies that the proper function $\lambda(x)$ is non-increasing along the trajectories of the controlled system, and it can be shown that this is sufficient to imply asymptotic stability of the controlled system.

Hence, by properly "scaling" the family of linear controllers (2), we have obtained a bounded globally asymptotically stabilizing feedback for (1). Based on homogeneity properties, the previous approach was generalized to any chain of integrators in [12]. The main contribution of this paper, is to show that we can in fact generalize it to any singleinput null-controllable system. The following section provides the general tool to do it. 


\section{$3 \quad$ Rescaling of control laws}

Consider a control system

$$
\dot{x}=f(x, u) \quad f \in \mathcal{C}^{1}\left(\mathbb{R}^{n} \times \mathbb{R}^{m} ; \mathbb{R}^{n}\right),
$$

with a one-parameter family of control laws $u(\lambda, x, t)$ and Lyapunov functions candidates $V(\lambda, x, t) \quad(\lambda \in \mathbb{R})$.

Assumption: There exists an interval $\Lambda=\left[\lambda_{0},+\infty\right)\left(\right.$ or $\left.\left(\lambda_{0},+\infty\right)\right)$ in $\mathbb{R}$ such that:

$A$. For any $\lambda \in \Lambda$, the feedback law $u(\lambda, x, t)$ makes the origin of the system (8) globally asymptotically stable.

$B$. For any $\lambda \in \Lambda$, the function $V(\lambda, x, t)$ is non-increasing along the trajectories of (8) controlled by $u(\lambda, x, t)$.

$C . u, V \in \mathcal{C}^{0}\left(\Lambda \times \mathbb{R}^{n} \times \mathbb{R} ; \mathbb{R}^{m}\right), V(\lambda, .,.) \in \mathcal{C}^{1}\left(\mathbb{R}^{n} \times \mathbb{R} ; \mathbb{R}\right)$ for any $\lambda \in \Lambda$, and both $u$ and $V$ are $T$-periodic with respect to $t$, piecewise $\mathcal{C}^{1}$ and everywhere right and left differentiable with respect to $\lambda$. For any $(\lambda, t) \in \Lambda \times \mathbb{R}, V(\lambda, ., t)$ is positive definite, proper, and vanishes at the origin. For any $(\lambda, t) \in \Lambda \times \mathbb{R}, u(\lambda, 0, t)=0$.

With this assumption we shall define a function $\lambda(x, t)$ which is equal to $\lambda_{0}$ at $x=0$, and is such that the feedback $u(\lambda(x, t), x, t)$ is still asymptotically stable for the system (8). More precisely, we have the following result which extends [9, Th. 4].

Theorem 1 Suppose that:

1. For any $(x, t), \lim V(\lambda, x, t)=0$ as $\lambda$ tends to $+\infty$, and $\lim V(\lambda, x, t)$ exists in $[0,+\infty]$ as $\lambda$ tends to $\lambda_{0}$, so that we can define a partition $\left(E_{0}, E_{1}\right)$ of $\mathbb{R}^{n} \times \mathbb{R}$ by:

$$
\begin{aligned}
& E_{0}=\left\{(x, t): \lim _{\lambda \longmapsto \lambda_{0}} V(\lambda, x, t) \leq 1\right\}, \\
& E_{1}=\left\{(x, t): \lim _{\lambda \longmapsto \lambda_{0}} V(\lambda, x, t)>1\right\} .
\end{aligned}
$$

2. $\lambda_{0} \notin \Lambda \Longrightarrow E_{0}=\{0\} \times \mathbb{R}$.

3. $V(\lambda, x, t)=1 \Longrightarrow \frac{\partial^{+}}{\partial \lambda} V(\lambda, x, t)<0$ and $\frac{\partial^{-}}{\partial \lambda} V(\lambda, x, t)<0$, with $\frac{\partial^{+}}{\partial \lambda}$ and $\frac{\partial^{-}}{\partial \lambda}$ the right and left derivatives with respect to $\lambda$.

Then,

i) For any $(x, t) \in E_{1}$, the equation

$$
V(\lambda, x, t)=1
$$

has a unique solution $\lambda \in \Lambda$. 
ii) The function $\lambda$ defined by

$$
\lambda(x, t)= \begin{cases}\lambda_{0} & \text { if }(x, t) \in E_{0}, \\ \text { the solution of }(10) & \text { if }(x, t) \in E_{1},\end{cases}
$$

is $\mathcal{C}^{0}$, Lipschitz continuous on $\mathbb{R}^{n} \times \mathbb{R}$ (resp. on $\left(\mathbb{R}^{n} \backslash\{0\}\right) \times \mathbb{R}$ ) if $\lambda_{0} \in \Lambda$ (resp. if $\left.\lambda_{0} \notin \Lambda\right)$, and T-periodic with respect to $t$.

iii) If $\lambda_{0} \in \Lambda$, the feedback law $u(x, t)$ defined by:

$$
u(x, t)=\left\{\begin{array}{cl}
0 & \text { if } x=0 \\
u(\lambda(x, t), x, t) & \text { otherwise }
\end{array}\right.
$$

is $\mathcal{C}^{0}$ and makes the origin of the system (8) globally asymptotically stable. If $\lambda_{0} \notin \Lambda$, $u(x, t)$ makes the origin of $(8)$ globally asymptotically stable provided that all solutions are well defined.

(Proof in Appendix)

\section{Remarks:}

1. Assumptions 1 and 2 imply that $E_{0}$ is always a closed set containing $\{0\} \times \mathbb{R}$, and therefore that $E_{1}$ is open.

2. The main assumption in this Theorem is Assumption 3 introduced in [9] in a different way as a "transversality condition".

3. If $\lambda_{0} \in \Lambda$, the feedback law (12) is continuous since both $(x, t) \longmapsto \lambda(x, t)$ and $(\lambda, x, t) \longmapsto u(\lambda, x, t)$ are continuous, and since $u(\lambda, 0, t) \equiv 0$ (Assumption C). If $\lambda_{0} \notin$ $\Lambda$, the feedback law (12) might be discontinuous at $x=0$. Indeed, $\lambda(x, t) \longrightarrow \lambda_{0}$ as $x \longrightarrow 0$, but $u(\lambda, x, t)$ might not have a continuous prolongation as $\lambda \longrightarrow \lambda_{0}$, and this can imply that $u(\lambda(x, t), x, t)$ does not tend to zero as $x \longrightarrow 0$. This is the reason why, in general, solutions from the origin might not be defined. In the applications that we consider here, this "pathological case" will never occur. Finally, note also that when $\lambda_{0} \notin \Lambda, \lim _{\lambda \rightarrow \lambda_{0}} V(\lambda, x, t)>1$ for any $t$ and $x \neq 0$. Since $V(\lambda, 0, t) \equiv 0, V$ can never be prolonged by continuity as $\lambda \longrightarrow \lambda_{0}$ in this case.

If $\lambda_{0} \notin \Lambda$, Assumption 2 implies that $E_{0}=\{0\} \times \mathbb{R}$. In this case Theorem 1 may be used to modify the asymptotic convergence rate, or to transform a non-robust feedback into a robust one. This will be briefly discussed in Section 4 .

If $\lambda_{0} \in \Lambda,(11)$ and (12) imply that the the " $\lambda$-constant" feedback $u\left(\lambda_{0}, x, t\right)$ is applied in a neighborhood of the origin (more precisely, in $E_{0}=\left\{(x, t): V\left(\lambda_{0}, x, t\right) \leq 1\right\}$ ) whereas the " $\lambda$-varying" feedback $u(\lambda(x, t), x, t)$ is applied outside this set. In this case, a possible application of Theorem 1 is to the problem of stabilization with control limitations, where one wants to satisfy some nominal/optimal behavior close to the equilibrium point, and rescale the controller when saturation problems may occur. This application will be discussed in Section 5. 


\section{Stabilization of homogeneous systems}

In this section, we briefly discuss some consequences of Theorem 1 for homogeneous control systems. The result given in this section is an extension of [9, Th. 4]. It also makes use of ideas developed in [12].

We consider systems homogeneous with respect to the linear Euler vector field (see e.g. [2] for more details). General definitions of homogeneity (with respect to nonlinear vector fields) can be found in the recent contributions [13] and [3] (see also [12] for applications). More precisely, given a set of real parameters $r_{i}>0(i=1, \ldots, n)$, a family of dilations (denoted as $\left(\delta_{\alpha}\right)$ ) is a family of applications $\delta_{\alpha}(\alpha>0)$ from $\mathbb{R}^{n}$ to $\mathbb{R}^{n}$ defined by $\delta_{\alpha} x=$ $\left(\alpha^{r_{1}} x_{1}, \ldots, \alpha^{r_{n}} x_{n}\right)$.

A continuous, $T$-periodic with respect to $t$ function $f$ is homogeneous of degree $\tau \geq 0$ with respect to the family of dilations $\left(\delta_{\alpha}\right)$ if:

$$
\forall t, \forall \alpha>0, \quad f\left(\delta_{\alpha} x, t\right)=\alpha^{\tau} f(x, t) .
$$

An homogeneous norm is any $\mathcal{C}^{0}$ function that is non-negative, proper, and degree one homogeneous.

A continuous, $T$-periodic with respect to $t$ vector field $f$ is homogeneous of degree $\tau$ with respect to the family of dilations $\left(\delta_{\alpha}\right)$ if, for any $i=1, \ldots, n$, the $i$-th component $f_{i}$ of $f$ is homogeneous of degree $\tau+r_{i}$.

The following is a consequence of Theorem 1 (compare with [9, Th. 4]).

Proposition 1 Consider the control system

$$
\dot{x}=f_{0}(x)+\sum_{i=1}^{m} u_{i} f_{i}(x)
$$

with $f_{k} \in \mathcal{C}^{1}\left(\mathbb{R}^{n} ; \mathbb{R}^{n}\right)(k=0, \ldots, m)$. Assume that:

1. The $\mathcal{C}^{0}$ and T-periodic with respect to $t$ feedback law $u(x, t)=\left(u_{1}(x, t), \ldots, u_{m}(x, t)\right)$ makes the origin of the system (13) globally asymptotically stable.

2. There exists a family of dilation $\left(\delta_{\alpha}\right)$ with respect to which each $f_{k}$ is homogeneous of degree $\tau_{k}$ with $\tau_{i}<\tau_{0}(i=1, \ldots, m)$, and $\tau_{0}=0$ if $u$ is time varying.

3. The $\mathcal{C}^{1}$ and T-periodic with respect to t function $V(x, t)$ (or $V(x)$ if $u$ is autonomous), with $V(0, t) \equiv 0$, is definite positive, proper with respect to $x$, non-increasing along the trajectories of the system (13) controlled by $u(x, t)$, and satisfies:

$$
\exists c>0: \quad \frac{\partial V}{\partial x}(x, t)\left(r_{1} x_{1}, \ldots, r_{n} x_{n}\right)>0 \quad \forall(x, t) \in V^{-1}(c) .
$$

Consider the feedback law $v(x, t)$ defined by

$$
\begin{aligned}
& v_{i}(0, t)=0 \\
& v_{i}(x, t)=\lambda^{\tau_{0}-\tau_{i}}(x, t) u_{i}\left(\delta_{\frac{1}{\lambda(x, t)}} x, t\right) \quad(x \neq 0),
\end{aligned}
$$

with $\lambda(x, t)$ solution of $c^{-1} V\left(\delta_{\frac{1}{\lambda}} x, t\right)=1$. Then, 
i) Each $v_{i}$ is continuous, $T$-periodic with respect to $t$ and homogeneous of degree $\tau_{0}-\tau_{i}$. It is Lipschitz continuous on $\left(\mathbb{R}^{n} \backslash\{0\}\right) \times \mathbb{R}$ if $u$ is Lipschitz continuous on this set.

ii) The system (13) controlled by the feedback law $v(x, t)$ is asymptotically stable and homogeneous of degree $\tau_{0}$. In particular, if $\tau_{0}=0$, it is $\rho$-exponentially stable, i.e. for any homogeneous norm $\rho$, there exist $K$ and $\gamma>0$ such that, along any solution of the system, $\rho(x(t)) \leq K \rho(x(0)) e^{-\gamma t}$.

Proof: First, for any $\lambda>0$, the control law

$$
u(\lambda, x, t)=\lambda^{\tau_{0}-\tau_{i}} u_{i}\left(\delta_{\frac{1}{\lambda}} x, \lambda^{\tau_{0}} t\right)
$$

is also an asymptotically stabilizing feedback for the system (13). This is easily shown, using the fact that each vector field $f_{k}$ is homogeneous of degree $\tau_{k}$, by taking the derivative of $y(t)=\delta_{\lambda} x\left(\lambda^{\tau_{0}} t\right)$ where $x($.$) is any solution of the system (13)$ controlled by $u(x, t)$. Similarly, one also easily shows that for any $\lambda>0$ and any $c>0$, the function

$$
V(\lambda, x, t)=c^{-1} V\left(\delta_{\frac{1}{\lambda}} x, \lambda^{\tau_{0}} t\right)
$$

is non-increasing along the trajectories of (13) controlled by (16). In view of Assumption 2 on $\tau_{0}$, we can rewrite $(16)$ as

$$
u(\lambda, x, t)=\lambda^{\tau_{0}-\tau_{i}} u_{i}\left(\delta_{\frac{1}{\lambda}} x, t\right)
$$

and in view of Assumption 3 on $V$, we can rewrite (17) as

$$
V(\lambda, x, t)=c^{-1} V\left(\delta_{\frac{1}{\lambda}} x, t\right)
$$

The families $u(\lambda, . .$.$) and V(\lambda, .,$.$) satisfy Assumptions A, B$, and $C$ of Section 3 with $\Lambda=(0,+\infty)$. One also easily verifies that Assumption 1 of Theorem 1 is satisfied with $E_{0}=\{0\} \times \mathbb{R}$. As a consequence, Assumption 2 is also satisfied. Finally, in view of (19), Assumption 3 requires

$$
V\left(\delta_{\frac{1}{\lambda}} x, t\right)=c \quad \Longrightarrow \frac{\partial}{\partial \lambda} V\left(\delta_{\frac{1}{\lambda}} x, t\right)<0
$$

Using the fact that

$$
\begin{aligned}
\frac{\partial}{\partial \lambda} V\left(\delta_{\frac{1}{\lambda}} x, t\right) & =\frac{\partial V}{\partial x}\left(\delta_{\frac{1}{\lambda}} x, t\right) \frac{\partial}{\partial \lambda} \delta_{\frac{1}{\lambda}} x \\
& =-\frac{1}{\lambda} \frac{\partial V}{\partial x}\left(\delta_{\frac{1}{\lambda}} x, t\right)\left(r_{1} \frac{1}{\lambda^{r_{1}}} x_{1}, \ldots, r_{n} \frac{1}{\lambda^{r_{n}}} x_{n}\right)
\end{aligned}
$$

it readily follows from (20) that Assumption 3 of Theorem 1 is precisely (14). By application of this theorem, $\lambda$ is continuous and $T$ periodic. In order to show that $v$ is continuous, one still needs to show, in view of $(15)$, that as $\lambda(x, t)$ tends to zero (i.e., as $x$ tends to zero), $v$ also tends to zero. Let us first show that for any $t, \lambda(., t)$ is homogeneous of degree 1 with respect to the family of dilation $\left(\delta_{\alpha}\right)$. Indeed, $\lambda\left(\delta_{\alpha} x, t\right)$ is defined by the equality

$$
c^{-1} V\left(\delta_{\frac{1}{\lambda\left(\delta_{\alpha} x, t\right)}} \delta_{\alpha} x, t\right)=1 .
$$


Since for any $\alpha_{1}$ and $\alpha_{2}, \delta_{\alpha_{1}} \delta_{\alpha_{2}} x=\delta_{\alpha_{1} \alpha_{2}} x,(22)$ implies that

$$
c^{-1} V\left(\delta_{\frac{\alpha}{\lambda(\delta \alpha x, t)}} x, t\right)=1 .
$$

Since $\lambda(x, t)$ is uniquely defined, it follows that $\frac{\alpha}{\lambda\left(\delta_{\alpha} x, t\right)}=\frac{1}{\lambda(x, t)}$ which precisely means that $\lambda$ is homogeneous of degree 1 . This readily implies that each $u_{i}\left(\delta_{\frac{1}{\lambda(x, t)}} x, t\right)$ is homogeneous of degree 0 and therefore, that each $v_{i}$ is homogeneous of degree $\tau_{0}-\tau_{i}>0$. This implies that $v_{i}$ tends to zero as $x$ tends to zero ${ }^{1}$. Thus, $v$ is continuous. The periodicity of $v_{i}$ with respect to $t$ follows from the periodicity of $\lambda$ and $u$. The Lipschitz continuity follows from that of $\lambda$ (Property ii) of Theorem 1) and that of $u$. This concludes the proof of i).

Now, we prove ii). Since the feedback law $v(x, t)$ is continuous, asymptotic stability of the origin of the closed-loop system follows readily by application of Theorem 1 . The fact that the closed loop system is homogeneous of degree $\tau_{0}$ is a direct consequence of i) and of Assumption 2. Finally, the $\rho$-exponential stability comes from the fact that the closed-loop system is degree zero homogeneous (see [3] for details).

\section{$5 \quad$ Stabilization with control limitations}

In this section, we consider the problem of stabilization with control limitations of the form $|u| \leq M$. We consider a single-input linear controllable system:

$$
\dot{x}=A x+b u \text {. }
$$

We assume throughout this section that $A$ is in companion form and $b=(0, \ldots, 0,1)^{T}$.

It is well known that a necessary and sufficient condition for the existence of a bounded, globally asymptotically stabilizing feedback for the system (23) is that $A$ has no eigenvalues with strictly positive real part. In the past few years, several methods have been developed in order to design such stabilizing feedbacks. Let us just mention the works of Teel and Sussmann and al. $[16,15]$ based on nested saturations, or the approach by Megretski, Lin, or Teel $[10,8,17]$ based on updating the solution of a Ricatti equation. We show in this section that Theorem 1 can also be used to construct bounded globally stabilizing feedbacks. As a matter of fact, our approach is strongly related to the recent result of Megretski [10]. More precisely, the condition $d P(r) / d r>0$ in the definition of an $(\mathcal{A}, B, W)$-chain in [10] is equivalent to the "transversality condition" (Assumption 3 of Theorem 1), and the matrix family $P(r) r \in[0,1]$ plays the role of our family of functions $V(\lambda, .,$.$) . By comparison$ with [10], the fact that we only require the Lyapunov function to be non-increasing will allow us to find an explicit family $V(\lambda, .,$.$) . However, as a counterpart, the stability and$ boundedness analysis is made much trickier.

When all eigenvalues of the matrix $A$ in (23) are zero (i.e., for a chain of integrators) Theorem 1 easily leads to bounded stabilizing feedbacks. Indeed, in this case one easily checks that both the drift and the control vector fields are homogeneous of degree $-\tau$ with respect to the family of dilation defined by $\delta_{\alpha} x=\left(\alpha^{n \tau} x_{1}, \alpha^{(n-1) \tau} x_{2}, \ldots, \alpha^{\tau} x_{n}\right)$. By application of Proposition 1 (in fact, one needs to slightly modify this proposition since

\footnotetext{
${ }^{1}$ Note that this is the only place where we have used the assumption $\tau_{i}<\tau_{0}$. If instead we have some $\tau_{i}=\tau_{0}$, then the corresponding control is bounded but is in general discontinuous at $x=0$.
} 
Assumption 2 implies that the homogeneity of the drift vector field is strictly larger than the homogeneity of the control vector fields; however this assumption is only used to ensure the continuity at the origin, and is therefore not necessary if one only needs homogeneity outside some neighborhood of the origin), it follows that if for some linear stabilizing controller $u=K x$, there exists an associated Lyapunov function $V(x)$ such that

$$
\frac{\partial V}{\partial x}(x) .\left(n \tau x_{1},(n-1) \tau x_{2}, \ldots, \tau x_{n}\right)^{T}<0 \quad \forall x \neq 0,
$$

then one can construct another stabilizing feedback $v$ homogeneous of degree $\tau-\tau=0$. Since any function homogeneous of degree 0 is bounded, this gives a bounded stabilizing feedback. It has been shown in [12] that one could always find a stabilizing feedback $K x$ together with an associated Lyapunov function $V$ such that the above inequality is satisfied. Unfortunately, this homogeneity-based argument cannot be used for systems other than a chain of integrators since a necessary and sufficient condition for the existence of a family of dilation with respect to which $A x$ is homogeneous of non-zero degree is that $A$ is nilpotent (i.e., all eigenvalues must be equal to zero).

The design and analysis of the control laws are presented in the next three subsections. These subsections can be summarized as follows. First, we construct a family of controllers $u(\lambda,$.$) for the system (23)$ together with a family of functions $V(\lambda,$.$) . In particular, these$ controllers and functions satisfy Assumptions $A, B$, and $C$ of Section 3. They are also endowed with many degrees of freedom. Then, by fixing some of these degrees of freedom, we show how to fulfill the three assumptions of Theorem 1. Therefore we obtain a nonlinear stabilizing feedback for (23). Finally, we show that this feedback is bounded, and how to modify this bound.

Note that our family of function $u(\lambda,$.$) will be explicitely defined. Therefore, the$ sole on-line computation shall consist in solving the implicit equation (10). This is to be compared with the results in $[10,8,17]$ where heavier on-line computations have to be performed.

\subsection{The families $u(\lambda,$.$) and V(\lambda,$.}

The design of these families is based on the properties of the so-called "Schwartz matrices". Some of these properties are recalled here. The reader can consult [11] for additional properties and applications.

Definition 1 Let $s \in \mathbb{R}^{n}$. The matrix $S(s)$ defined by

$$
S(s)=\left(\begin{array}{cccccc}
0 & 1 & 0 & \ldots & \ldots & 0 \\
-s_{1} & 0 & 1 & 0 & \ldots & 0 \\
0 & -s_{2} & 0 & 1 & \ldots & 0 \\
0 & 0 & \ddots & \ddots & \ddots & 0 \\
0 & 0 & 0 & -s_{n-2} & 0 & 1 \\
0 & 0 & 0 & 0 & -s_{n-1} & -s_{n}
\end{array}\right)
$$

is called the "Schwartz matrix" associated with s. 
These matrices have important properties for control applications, some of which are now recalled.

Lemma 1 Let $u(x)=K x$ be any linear stabilizing feedback for (23). Then, there exist a vector $s \in \mathbb{R}^{n}$ with $s_{i}>0(i=1, \ldots, n)$, and a linear change of coordinates $x \longmapsto y=\xi(s) x$ which transforms the controlled system (23) into the Schwartz system

$$
\dot{y}=S(s) y \text {. }
$$

Conversely, for any vector $s \in \mathbb{R}^{n}$ with $s_{i}>0(i=1, \ldots, n)$, there exists a linear change of coordinates $y \longmapsto x=\psi(s) y$ which transforms the system (25) into the asymptotically stable system

$$
\dot{x}=A x+b K^{T}(s) x, \quad K^{T}(s)=\psi_{n}(s) S(s) \psi^{-1}(s)-A_{n} .
$$

Moreover,

i) The function $x^{T} \xi^{T}(s) D(s) \xi(s) x$, with

$$
D(s)=\operatorname{Diag}\left(\prod_{k=i}^{n-1} s_{k}\right),
$$

is non-increasing, and tends to zero, along the trajectories of (26).

ii) $\psi(s)$ and $\xi(s)$ are lower triangular matrices with the following form (similarly for $\xi(s))$

$$
\psi(s)=\left(\begin{array}{cccccc}
1 & 0 & \cdots & \ldots & \cdots & 0 \\
0 & 1 & \ddots & & & \vdots \\
\star & 0 & 1 & \ddots & & \vdots \\
0 & \star & 0 & 1 & \ddots & \vdots \\
\star & 0 & \star & 0 & 1 & 0 \\
& \ddots & \ddots & \ddots & \ddots & \ddots
\end{array}\right)
$$

More formally, $\psi_{i, j}(s)=\xi_{i, j}(s)=0$ for any $j \notin I_{i} \triangleq\{j \in \mathbb{N}: j \leq i$ and $i-j$ is even $\}$. The $\star$ 's are defined via the following relations:

$$
\begin{aligned}
& \left\{\begin{array}{lll}
\psi_{i, 1}(s)=-s_{1} \psi_{i-1,2}(s) & & (i \text { odd }, i>1) \\
\psi_{i, j}(s)=\psi_{i-1, j-1}(s)-s_{j} \psi_{i-1, j+1}(s) & & \text { otherwise, }
\end{array}\right.
\end{aligned}
$$

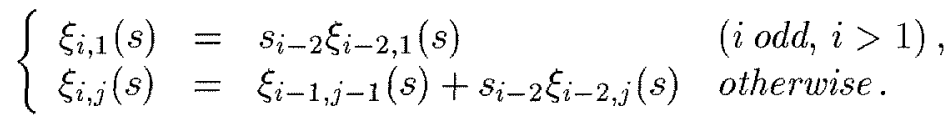

Proof: Part i) is a direct consequence of the fact that the function $y^{T} D(s) y$ is nonincreasing along the trajectories of the system (25) (its time derivative is $-2 s_{n} y_{n}^{2}$ ) and, by application of LaSalle's Theorem [5], converges to zero along these trajectories. The other 
properties can be checked easily by hand. For proofs, see [7] and [14] (see in particular [7, Eq. (10)] for (29) and [14, Eq. (21)] for (28)).

\section{Remarks:}

1. Given a stabilizing controller for (23), there is a systematic way to compute the vector $s$ such that (26) is transformed into (25): the components of $s$ are obtained from the first column of the Routh table (see e.g. [1, Sec. 8-3,8-5], [11]).

2. It is also shown in [14] that there exists a change of coordinates which transforms the control system (23) into a system in Schwartz form $\dot{y}=S(s) y+b u$. If $A$ is not Hurwitz, the vector $s$ is not unique in the sense that this transformation exists for any $s$ such that the characteristic polynomial of $S(s)$ is equal to the characteristic polynomial of $A$.

Lemma 1 provides us with families $u(\lambda,$.$) and V(\lambda,$.$) . Indeed, let us define the set \Lambda$ as

$$
\Lambda=[1,+\infty) .
$$

We also consider any vector valued function $s: \Lambda \longmapsto \mathbb{R}_{+}^{n}$ and any function $k: \Lambda \longmapsto \mathbb{R}_{+}$ with $s$ and $k$ being $\mathcal{C}^{0}$, piecewise $\mathcal{C}^{1}$ and everywhere left and right differentiable. We define the family $u(\lambda,$.$) by:$

$$
u(\lambda, x) \triangleq K^{T}(\lambda) x \triangleq[\psi(s(\lambda)) S(s(\lambda)) \xi(s(\lambda))-A]_{n} x,
$$

and the family $V(\lambda,$.$) by:$

$$
V(\lambda, x) \triangleq k(\lambda) x^{T} \xi^{T}(s(\lambda)) D(s(\lambda)) \xi(s(\lambda)) x .
$$

Then, it immediately follows from Lemma 1 that Assumptions $A, B$, and $C$ of Section 3 are satisfied for the system (23). Therefore, for any choice of $s$ and $k$ such that the assumptions of Theorem 1 are satisfied, the controller $u(\lambda(x), x)$ with $u(\lambda, x)$ defined by $(31)$, and $\lambda(x)$ defined by

$$
\lambda(x)= \begin{cases}1 & \text { if } V(1, x) \leq 1, \\ \text { the solution of } V(\lambda, x)=1 & \text { otherwise }\end{cases}
$$

ensures global asymptotic stability of (23).

\subsection{The stability conditions}

In this section we provide a specific vector-valued function $s$, and a function $k$ in order to fulfill the assumptions of Theorem 1 . We will show in the next subsection that the non-linear controller (12) obtained by application of this theorem solves our problem of stabilization with bounded control.

First, (see Remark 2 in Section 5.1) the system (23) can be rewritten, after some possible change of coordinates $x \longmapsto \tilde{x}$ into the Schwartz representation:

$$
\dot{\tilde{x}}=S\left(c_{0}\right) \tilde{x}+b u,
$$

with

$$
c_{0}=(\underbrace{0, \ldots, 0}_{m}, \underbrace{\omega_{1}^{2}, 0, \ldots, \omega_{p}^{2}, 0}_{2 p}, \underbrace{c_{0, m+2 p+1}, \ldots, c_{0, n}}_{q})^{T} .
$$


$m$ is the number of 0 eigenvalues, $2 p$ is the number of pure imaginary eigenvalues, and $q=n-m-2 p$ is the number of eigenvalues with strictly negative real part. Each $\pm j \omega_{i}$ is an imaginary eigenvalue of $A$, and the $c_{0, k}>0(k=m+2 p+1, \ldots, n)$ are associated with the stable part of $A$. More precisely, let $P_{1}(x) P_{2}(x)$ be the factorization of the characteristic polynomial of $A$ into a Hurwitz and non-Hurwitz polynomial, each $\pm j \omega_{i}$ can be obtained as a zero of $P_{2}$, and the $c_{0, k}(k=m+2 p+1, \ldots, n)$ can be computed from the Routh-Hurwitz table associated with $P_{1}$.

The functions $k$ and $s_{i}$ are defined by:

$$
\begin{gathered}
k(\lambda)= \begin{cases}\eta \lambda^{-2 \beta} & \lambda \in\left[1, \lambda_{1}\right] \\
\eta \lambda_{1}^{2(1-\beta)} \lambda^{-2} & \lambda \in\left[\lambda_{1},+\infty\right),\end{cases} \\
s_{i}(\lambda)= \begin{cases}\frac{c_{1, i}}{\lambda^{2 \tau_{1, i}}} & \lambda \in\left[1, \lambda_{1}\right] \\
\frac{c_{2, i}}{\lambda^{2 \tau_{2, i}}}\left(\frac{\lambda_{1}}{\lambda_{2}}\right)^{2 \tau_{2, i}} & \lambda \in\left[\lambda_{1},+\infty\right) .\end{cases}
\end{gathered}
$$

The various parameters in (36) and (37) are to be chosen as follows:

$\eta>0$ is used to modify the bound satisfied by the control law. The relations between its value and this bound will be studied in the next section.

$\lambda_{1}>1$.

$c_{1, i}>0(i=1, \ldots, n)$. These coefficients define the (linear) controller applied in a neighborhood of the origin.

The parameters $\tau_{1, i}$ are defined in order to ensure the continuity of the functions $s_{i}$ at $\lambda=\lambda_{1}$. More precisely, we define

$$
\tau_{1, i}=\frac{1}{2 \ln \lambda_{1}}\left(\ln c_{1, i}-\ln c_{2, i}+2 \tau_{2, i} \ln \lambda_{2}\right)
$$

The parameters $\tau_{2, i}$ are chosen to guarantee the boundedness of the controller (this is shown in Section 5.3), and also to ensure in part that Assumption 3 of Theorem 1 is satisfied,

$$
\tau_{2, i}=\left\{\begin{array}{cl}
0 & \text { if } c_{0, i} \neq 0 \\
\frac{1}{2} & \text { if } c_{0, i}=0 \text { and } i=n \\
1+\sum_{k=i+1}^{n-1} \tau_{2, k} & \text { if } c_{0, i}=0 \text { and } m-1 \leq i \leq n-1 \\
\tau_{2, m-1} & \text { if } c_{0, i}=0 \text { and } i \leq m-2
\end{array}\right.
$$

The parameters $c_{2, i}$ are also chosen in order to guarantee Assumption 3 (for $\lambda \geq \lambda_{1}$ ). We show bellow that this assumption is equivalent here to

$$
D(\lambda)\left[-R+\lambda \frac{\partial \xi}{\partial \lambda}(\lambda) \psi(\lambda)\right]<0
$$


with

$$
R=\operatorname{Diag}\left(r_{i}\right), \quad r_{i}=1+\sum_{k=i}^{n-1} \tau_{2, i} .
$$

To get (40), we choose:

$$
\begin{cases}c_{2, i}=c_{0, i} & \text { if } c_{0, i} \neq 0 \\ c_{2, i}>0 & \text { if } c_{0, i}=0 \text { and } m-1<i\end{cases}
$$

and for $i=1, \ldots, m-1, c_{2, i}$ is recursively defined as any solution of the following LMI in $\frac{1}{c_{2, i}}$ :

$$
C^{i+1} G^{i+1}=\frac{1}{c_{2, i}}\left(\begin{array}{ccc}
0 & 0 & 0 \\
C_{i, i} G_{i+1,1} & \ldots & C_{i, i} G_{i+1, i+1}
\end{array}\right)+\left(\begin{array}{cc}
C^{i} G^{i} & 0 \\
0 & 0
\end{array}\right)<0
$$

with

$$
\begin{aligned}
& C=\operatorname{Diag}\left(\prod_{k=1}^{i-1} \frac{1}{c_{2, k}}\right), \quad G=-R+\left[\frac{\partial \xi(\tilde{s}(\tilde{\lambda}))}{\partial \tilde{\lambda}} \psi(\tilde{s}(\tilde{\lambda}))\right]_{\mid \tilde{\lambda}=1}, \\
& \tilde{s}(\tilde{\lambda})=\left(\frac{c_{2,1}}{\tilde{\lambda}^{2 \tau_{2,1}}}, \ldots, \frac{c_{2, n}}{\tilde{\lambda}^{2 \tau_{2, n}}}\right) .
\end{aligned}
$$

All parameters have been specified but $\beta$ and $\lambda_{2}$. These must be chosen in accordance with the following proposition.

Proposition 2 For any choice of the above parameters, Assumptions 1 and 2 of Theorem 1 are satisfied and,

i) There exists $\bar{\lambda}_{2}$ such that for $\lambda_{2}>\bar{\lambda}_{2}$, Assumption 3 of Theorem 1 is satisfied for $\lambda \in\left[\lambda_{1},+\infty\right)$. In particular, if all eigenvalues of $A$ are zero (i.e., for a chain of integrators), Assumption 3 is satisfied for any $\lambda_{2}>0$.

ii) For any $\lambda_{2}$, there exists $\beta\left(\lambda_{2}\right)$ such that for $\beta>\beta\left(\lambda_{2}\right)$, Assumption 3 of Theorem 1 is satisfied for $\lambda \in\left[1, \lambda_{1}\right]$.

Before giving the proof of this proposition, let us make some remarks on the choice of the different control parameters.

1. When the control parameters are chosen as indicated in Proposition 2, Theorem 1 applies to yield the stabilizing controller (31)-(33). This controller has the following characteristics.

On the set $E_{0}=\{x: V(1, x) \leq 1\}$, the function $\lambda(x)$ defined by (33) is identically equal to 1 . Therefore, the controller (31) will be linear on this set. Moreover, in view of $(37), s_{i}(1)=c_{1, i}$. In view of Lemma 1 , it follows that any linear controller can be applied by choosing the corresponding $c_{1, i}$.

On the set $\left\{x: V\left(\lambda_{1}, x\right) \geq 1\right\}=\left\{x: \lambda(x) \geq \lambda_{1}\right\}$, the function $x$ is unbounded. Therefore, on this set the control limitations will become predominant. In view of (37), the parameters $c_{2, i}$ and $\tau_{2, i}$ must be chosen in order to be able to satisfy these limitations. In particular, in view of $(37),(39)$, and (42), each function $s_{i}(\lambda)$ tends to the coefficient $c_{0, i}$ of the open-loop system (34) as $\lambda$ tends to $+\infty$. 
The set $\left\{x: 1<\lambda(x)<\lambda_{1}\right\}$ is only used to make the transition between the two previous sets. To put it in another way, it makes the transition between a local controller designed in order to satisfy some performance criteria, and a global controller designed in order to ensure control limitations.

2. If one is only interested in semi-global stability instead of global stability, one can basically neglect the definition of the function $s$ on the interval $\left[\lambda_{1},+\infty\right)$, and most of the design complexity is then avoided. By doing so, the feedback law will ensure asymptotic stability for any trajectory starting from $\left\{x: V\left(\lambda_{1}, x\right) \leq 1\right\}$ and for any choice of the $\tau_{1, i}$ provided that $\beta$ is chosen large enough. In this case, the $\tau_{1, i}$ should be chosen large enough, so that the control law satisfies the control limitation on $\left\{x: V\left(\lambda_{1}, x\right) \leq 1\right\}$.

Proof: In view of (36) and (32), the function $V$ is defined by

$$
V(\lambda, x)=x^{T} P(\lambda) x
$$

with

$$
P(\lambda)= \begin{cases}\eta \lambda^{-2 \beta} \xi^{T}(s(\lambda)) D(s(\lambda)) \xi(s(\lambda)) & \lambda \in\left[1, \lambda_{1}\right] \\ \eta \lambda_{1}^{2(1-\beta)} \lambda^{-2} \xi^{T}(s(\lambda)) D(s(\lambda)) \xi(s(\lambda)) & \lambda \in\left[\lambda_{1},+\infty\right)\end{cases}
$$

In the sequel, we will often make the following abuses of notations: $\psi(\lambda) \triangleq \psi(s(\lambda)), \xi(\lambda) \triangleq$ $\xi(s(\lambda))$, and $D(\lambda) \triangleq D(s(\lambda))$. It is immediate to verify that $V$ satisfies the continuity and differentiability assumptions of Theorem 1 . Moreover, $\lim _{\lambda \mapsto+\infty} V(\lambda, x)=0$ because each function $s_{i}$ is non-increasing in $\lambda$ for $\lambda \in\left[\lambda_{1},+\infty\right)$ (see (37)-(39)) and, in view of (27)-(28), the matrices $\xi(s)$ and $D(s)$ are polynomial in the $s_{i}$ 's. As a consequence, Assumptions 1 and 2 of Theorem 1 are satisfied.

We now consider Assumption 3. Henceforth, we denote ' the derivative with respect to $\lambda$. Using (27)-(37), one easily shows that

$$
D^{\prime}(\lambda)= \begin{cases}-\frac{2}{\lambda} D(\lambda)(Q-\operatorname{Id}(n)) & \lambda \in\left[1, \lambda_{1}\right] \\ -\frac{2}{\lambda} D(\lambda)(R-\operatorname{Id}(n)) & \lambda \in\left[\lambda_{1},+\infty\right),\end{cases}
$$

with $R$ defined by (41) and $Q$ defined by

$$
Q=\operatorname{Diag}\left(q_{i}\right), \quad q_{i}=1+\sum_{k=i}^{n-1} \tau_{1, i}
$$

Since $\xi=\psi^{-1}$, Assumption 3 of Theorem 1 can be rewritten, from (45), (46), and (47), as

$$
\begin{array}{ll}
2 \eta \lambda^{-(2 \beta+1)} \xi^{T}(\lambda) D(\lambda)\left[-(\beta-1) \operatorname{Id}(n)-Q+\lambda \xi^{\prime} \psi(\lambda)\right] \xi(\lambda)<0 & \lambda \in\left[1, \lambda_{1}\right] \\
2 \eta \lambda_{1}^{2(1-\beta)} \lambda^{-3} \xi^{T}(\lambda) D(\lambda)\left[-R+\lambda \xi^{\prime} \psi(\lambda)\right] \xi(\lambda)<0 & \lambda \in\left[\lambda_{1},+\infty\right) .
\end{array}
$$

Consider first the case $\lambda \in\left[1, \lambda_{1}\right]$. Then, (49) is equivalent to

$$
D(\lambda)\left[-(\beta-1) \operatorname{Id}(n)-Q+\lambda \xi^{\prime} \psi(\lambda)\right]<0, \quad \lambda \in\left[1, \lambda_{1}\right] .
$$

In view of (27) and (37), $D(\lambda)>0$. Moreover, $D(\lambda), Q$, and $\lambda \xi^{\prime}(\lambda) \psi(\lambda)$ are only functions of the $s_{i}(\lambda)$ and $\tau_{1, i}$. Therefore, they are independent of $\beta$ and bounded on the compact 
set $\left[1, \lambda_{1}\right]$. This immediately implies that $(50)$ is satisfied for $\beta$ large enough, and part ii) of the proposition is proved.

Now we assume that $\lambda \in\left[\lambda_{1},+\infty\right)$. (49) becomes

$$
D(\lambda)\left[-R+\lambda \xi^{\prime} \psi(\lambda)\right]<0, \quad \lambda \in\left[\lambda_{1},+\infty\right) .
$$

In view of $(27),(41)$, and (37),

$$
D(\lambda)=\operatorname{Diag}\left(\left(\frac{\lambda \lambda_{2}}{\lambda_{1}}\right)^{-2\left(r_{i}-1\right)}\right) \operatorname{Diag}\left(\prod_{k=i}^{n-1} c_{2, k}\right) .
$$

As a consequence,

$$
D(\lambda)\left[-R+\lambda \xi^{\prime} \psi(\lambda)\right]=\operatorname{Diag}\left(\left(\frac{\lambda \lambda_{2}}{\lambda_{1}}\right)^{-r_{i}+1}\right) N(\lambda) \operatorname{Diag}\left(\left(\frac{\lambda \lambda_{2}}{\lambda_{1}}\right)^{-r_{i}+1}\right),
$$

with

$$
N(\lambda)=\operatorname{Diag}\left(\left(\frac{\lambda \lambda_{2}}{\lambda_{1}}\right)^{-r_{i}+1}\right) \operatorname{Diag}\left(\prod_{k=i}^{n-1} c_{2, k}\right)\left[-R+\lambda \xi^{\prime} \psi(\lambda)\right] \operatorname{Diag}\left(\left(\frac{\lambda \lambda_{2}}{\lambda_{1}}\right)^{r_{i}-1}\right)
$$

Furthermore, by making the change of variable $\tilde{\lambda}=\frac{\lambda \lambda_{2}}{\lambda_{1}}$, one easily obtains that

$$
N(\lambda)=\tilde{N}(\tilde{\lambda}) \triangleq \operatorname{Diag}\left(\tilde{\lambda}^{-r_{i}+1}\right) \operatorname{Diag}\left(\prod_{k=i}^{n-1} c_{2, k}\right)\left[-R+\tilde{\lambda} \tilde{\xi}^{o} \tilde{\psi}(\tilde{\lambda})\right] \operatorname{Diag}\left(\tilde{\lambda}^{r_{i}-1}\right)
$$

with

$$
\tilde{\psi}(\tilde{\lambda})=\psi(\tilde{s}(\tilde{\lambda})), \tilde{\xi}(\tilde{\lambda})=\xi(\tilde{s}(\tilde{\lambda})),
$$

$\tilde{s}$ defined by (44), and ${ }^{\circ}$ denoting the derivative with respect to $\tilde{\lambda}$. From (53) and (55), (51) is equivalent to

$$
\tilde{N}(\tilde{\lambda})<0, \quad \tilde{\lambda} \in\left[\lambda_{2},+\infty\right) \text {. }
$$

Moreover, we have

Lemma 2 Each component $\tilde{n}_{i, j}$ of $\tilde{N}$ satisfies

i) $\tilde{n}_{i, j}=0 \quad \forall j \notin I_{i}$ (recall that $I_{i}$ is defined in Lemma 1),

ii) $\tilde{n}_{i, i}=-r_{i} \prod_{k=i}^{n-1} c_{2, k}$,

iii) $\prod_{k=i}^{n-1} \frac{1}{c_{2, k}} \tilde{n}_{i, j}$ does not depend on the $c_{2, k}: k \geq i-1$,

iv) for $i \in\{1, \ldots, m\}, \tilde{n}_{i, j}$ is constant with respect to $\tilde{\lambda}$,

v) for $i \in\{m+1, \ldots, n\}$ and $j \neq i, \tilde{n}_{i, j}(\tilde{(}(\lambda))$ tends to zero as $\tilde{\lambda}$ tends to $+\infty$. 
(Proof in Appendix)

From this lemma, it is easy to conclude the proof. From i), ii), and iv), the upper left minor $\tilde{N}^{m}$ and all diagonal terms are independent of $\tilde{\lambda}$. From i) and v), all other terms tend to zero as $\tilde{\lambda}$ tends to $+\infty$. Since from ii), all diagonal terms are strictly negative, $(57)$ will be satisfied, for $\lambda_{2}$ large enough, if $\tilde{N}^{m}$ is negative definite. We claim that this is true when the coefficients $c_{2, i}(i=1, \ldots, m)$ are chosen according to (43). First, it follows from (55) and (44) that

$$
\tilde{N}(1)=\operatorname{Diag}\left(\prod_{k=i}^{n-1} c_{2, k}\right)\left(-R+\tilde{\xi}^{o} \tilde{\psi}(1)\right)=\left(\prod_{k=1}^{n-1} c_{2, k}\right) C G .
$$

Since $\tilde{N}^{m}$ is independent of $\tilde{\lambda}$,

$$
\tilde{N}^{m}=\tilde{N}^{m}(1)=\left(\prod_{k=1}^{n-1} c_{2, k}\right)(C G)^{m}=\left(\prod_{k=1}^{n-1} c_{2, k}\right) C^{m} G^{m},
$$

so that $\tilde{N}^{m}>0 \Longleftrightarrow C^{m} G^{m}>0$ which is precisely (43) for $i=m-1$. We still need to prove that (43) is solvable. We proceed by induction on $i$. We first remark that $C^{1} G^{1}<0$ since, in view of $(58), C^{1} G^{1}=\left(\prod_{k=1}^{n-1} \frac{1}{c_{2, k}}\right) \tilde{N}^{1}$ and, in view of Lemma $2, \tilde{N}^{1}=\tilde{n}_{1,1}=$ $-r_{1}<0$. Assume that (43) is satisfied up to $i>0$ and consider the matrix $C^{i+1} G^{i+1}$. From $(58), G^{i+1}=\operatorname{Diag}\left(\prod_{k=j+1}^{n-1} \frac{1}{c_{2, k}}\right) \tilde{N}^{i+1}$. Therefore, iii) in Lemma 2 implies that $G^{i+1}$ is independent of $c_{2, i}$, and so are $G^{i}$ and $G_{i+1}$. Since $C^{i}$ is also independent of $c_{2, i}$, we deduce that both $C^{i} G^{i}$ and $C_{i, i} G_{i+1}$ are independent of $c_{2, i}$. By the induction assumption $C^{i} G^{i}<0$, and $C_{i, i} G_{i+1, i+1}<0$ (it is equal to $-C_{i, i} r_{i+1}$ ). Since, from (43), $C^{i+1} G^{i+1}$ is bloc lower diagonal, i.e.

$$
C^{i+1} G^{i+1}=\left[\begin{array}{c|c}
C^{i} G^{i} & 0 \\
\hline \star & \frac{C_{i, i} G_{i+1, i+1}}{c_{2, i}}
\end{array}\right]
$$

it is easy to verify that (43) is satisfied for $c_{2, i}$ large enough. This concludes the proof.

Proposition 2 does not provide values $\bar{\lambda}_{2}$ and $\beta\left(\bar{\lambda}_{2}\right)$ for which the stability conditions are guaranteed. Obtaining the optimal (minimal) values seems particularly difficult. Yet, we can provide sufficient values of these parameters (note however that these values might be very conservative).

First, if $\lambda \in\left[1, \lambda_{1}\right]$, the condition to satisfy is (50). One easily verifies that this condition is satisfied if

$$
-((\beta-1) \operatorname{Id}(n)+Q) D_{\min }+D_{\max }\left(\lambda \xi^{\prime} \psi\right)_{\max }<0
$$

with:

$$
\begin{aligned}
& D_{\text {min }}=\operatorname{Diag}\left(\prod_{k=i}^{n-1} s_{k, \text { min }}\right), \quad s_{k, \text { min }}=\operatorname{Min}\left\{s_{k}(\lambda): \lambda \in\left[1, \lambda_{1}\right]\right\}=\operatorname{Min}\left(s_{k}(1), s_{k}\left(\lambda_{1}\right)\right) \\
& D_{\max }=\operatorname{Diag}\left(\prod_{k=i}^{n-1} s_{k, \max }\right), \quad s_{k, \max }=\operatorname{Max}\left\{s_{k}(\lambda): \lambda \in\left[1, \lambda_{1}\right]\right\}=\operatorname{Max}\left(s_{k}(1), s_{k}\left(\lambda_{1}\right)\right), \\
& \left(\lambda \xi^{\prime} \psi\right)_{\max }=W\left(s_{\max }\right),
\end{aligned}
$$


and $W(s)$ the matrix obtained from $\lambda \xi^{\prime}(s) \psi(s)$ by changing each monomial in $s$ into its absolute value. Remark that $\lambda \xi^{\prime}(s) \psi(s)$ can be expressed as a function of the $s_{i}$ since each $\xi_{i, j}$ and $\psi_{i, j}$ is polynomial in the $s_{i}$ 's and each $s_{i}$ is a fraction $\frac{c}{\lambda^{\tau}}$. In fact, we can obtain the following recursive definition of $\lambda \xi^{\prime}(s) \psi(s)$ as a polynomial matrix in the $s_{i}$ :

$$
\sigma_{i, j} \triangleq \lambda\left(\xi^{\prime} \psi\right)_{i, j}(s)= \begin{cases}0 & \left(j \notin I_{i}, \text { or } j=i\right) \\ \sigma_{i-1, j-1}-2 \tau_{j} s_{j} & (j=i-2) \\ \sigma_{i-1, j-1}-s_{j} \sigma_{i-1, j+1}+s_{i-2} \sigma_{i-2, j} & \text { otherwise }\end{cases}
$$

with $\tau_{j}$ in the second line of (63) equal to $\tau_{1, j}$ if $\lambda \in\left[1, \lambda_{1}\right)$, and to $\tau_{2, j}$ if $\lambda \in\left(\lambda_{1},+\infty\right)$. Finding some $\beta$ such that (61) is satisfied makes no difficulty since each matrix in (61) is now constant (i.e., independent of $\lambda$ ).

If $\lambda \in\left[\lambda_{1},+\infty\right)$, the condition to satisfy is (57). From the proof of Proposition 2,

$$
\tilde{N}(\tilde{\lambda})=E+F(\tilde{\lambda})
$$

with $E<0$ the constant part of $\tilde{N}$, and

$$
F(\tilde{\lambda})=\left(\begin{array}{c}
0 \\
F_{m+1}(\tilde{\lambda}) \\
\vdots \\
F_{n}(\tilde{\lambda})
\end{array}\right), \quad F_{i}(\tilde{\lambda})=\left(\tilde{n}_{i, 1}, \ldots, \tilde{n}_{i, i-1}\right) \quad(i>m) .
$$

Moreover, in view of the proof of Lemma 2 (in Appendix), we know that each $F_{i, j}$ is a sum of monomials in $\tilde{\lambda}$ of degree not larger than $-r_{i}$. As a consequence, using (65) and (55), we have:

$$
\begin{aligned}
\left|F_{i, j}(\tilde{\lambda})\right| & =\tilde{\lambda}^{-r_{i}}\left|\tilde{\lambda}^{r_{i}} F_{i, j}(\tilde{\lambda})\right|=\tilde{\lambda}^{-r_{i}}\left|\tilde{\lambda}^{-r_{j}}\left(\prod_{k=i}^{n-1} c_{2, k}\right)\left(\tilde{\lambda} \tilde{\xi} \tilde{\xi}^{o} \tilde{\psi}\right)_{i, j}(\tilde{\lambda})\right| \\
& \leq \tilde{\lambda}^{-r_{i}}\left(\tilde{\lambda}^{-r_{j}}\left(\prod_{k=i}^{n-1} c_{2, k}\right) w_{i, j}(\tilde{s}(\tilde{\lambda}))\right) \leq \tilde{\lambda}^{-r_{i}}\left(\prod_{k=i}^{n-1} c_{2, k}\right) w_{i, j}(\tilde{s}(1)) \quad \text { for } \tilde{\lambda} \geq 1,
\end{aligned}
$$

with $w_{i, j}(s)$ the components of the matrix $W(s)$ defined above. In view of $(64)$ and $(66)$, we have, for any $\tilde{\lambda} \geq 1$,

$$
x^{T} \tilde{N}(\tilde{\lambda}) x \leq \frac{1}{2} \sum_{i=m+1}^{n} \sum_{j=1}^{i-1}\left(x_{i}^{2}+x_{j}^{2}\right) \tilde{\lambda}^{-r_{i}}\left(\prod_{k=i}^{n-1} c_{2, k}\right) w_{i, j}(\tilde{s}(1))+x^{T} E x .
$$

Since the matrix associated with the right hand side of $(67)$ is monotonic in $\tilde{\lambda}$ (because each $w_{i, j}$ is positive), one easily finds some $\tilde{\lambda}_{2}$ for which (57) is satisfied.

\subsection{Boundedness of the controller}

We now assume that the functions $s_{i}$ defined by (37) have been chosen as indicated in the previous section. We consider the nonlinear globally asymptotically stabilizing feedback $u(x)$ defined by (12). Summarizing the results of Sections 5.1 and 5.2 , we have

$$
u(x)=K^{T}(\lambda(x)) x,
$$


with

$$
K^{T}(\lambda)=[\psi(s(\lambda)) S(s(\lambda)) \xi(s(\lambda))-A]_{n},
$$

and $\lambda(x)$ defined by

$$
\lambda(x)= \begin{cases}1 & \text { if } V(1, x) \leq 1 \\ \text { the solution of } V(\lambda, x)=1 & \text { otherwise }\end{cases}
$$

Recall that $\psi(s), S(s)$, and $\xi(s)$ are defined by (28), (24), and (29), and $s(\lambda), V(\lambda, x)$ are defined by (37), and (32). We have:

Proposition 3 The stabilizing feedback $u(x)$ defined by (68) is bounded and the bound is proportional to $\eta^{-\frac{1}{2}}$ (with $\eta$ defined in (36)).

Proof: First, let us recall that

$$
\operatorname{Max}\left\{\left|K^{T} x\right|: x^{T} P x=1\right\}=\left(K^{T} P^{-1} K\right)^{\frac{1}{2}} .
$$

Consider the matrix $P(\lambda)$ associated with the quadratic function $V(\lambda, x)$ (P is defined by (46)). Then, (71) and (68) imply that

$$
M(\lambda) \triangleq \operatorname{Max}\{|u(x)|: \lambda(x)=\lambda\}=\left(K^{T}(\lambda) P^{-1}(\lambda) K(\lambda)\right)^{\frac{1}{2}} .
$$

This is obviously true if $\lambda \in(1,+\infty)$ since $\lambda(x)=\lambda \Longleftrightarrow x^{T} P(\lambda) x=1$. This is also true for $\lambda=1$ since $\lambda(x)=1 \Longleftrightarrow x^{T} P(1) x \leq 1$. In view of (46) and (72), $M(\lambda)$ is proportional to $\eta^{-\frac{1}{2}}$. There remains to prove that $M(\lambda)$ is bounded on $[1,+\infty)$. We will prove that $M$ is bounded on $\left[\lambda_{1},+\infty\right)$ which is clearly sufficient since $M$ is continuous. We first note that the vector $K^{T}(\lambda)$ can be rewritten as

$$
K^{T}(\lambda)=\psi_{n}(s(\lambda)) S(s(\lambda)) \xi(s(\lambda))-\psi_{n}\left(c_{0}\right) S\left(c_{0}\right) \xi\left(c_{0}\right) .
$$

This comes from the fact that by definition, $\psi\left(c_{0}\right)$ is precisely the linear change of coordinates that transforms the open-loop system in Schwartz form (34) into the open-loop system in canonical form (23), so that $A_{n}=\psi_{n}\left(c_{0}\right) S\left(c_{0}\right) \xi\left(c_{0}\right)$. Let us now consider the following decomposition of $K^{T}(\lambda)$ :

$$
K^{T}(\lambda)=\left[K_{1}^{T}(\lambda)+K_{2}^{T}(\lambda)+K_{3}^{T}(\lambda)\right] \xi(s(\lambda)),
$$

with

$$
\begin{aligned}
& K_{1}^{T}(\lambda)=\psi_{n}(s)\left(S(s)-S\left(c_{0}\right)\right) \\
& K_{2}^{T}(\lambda)=\left(\psi_{n}(s)-\psi_{n}\left(c_{0}\right)\right) S\left(c_{0}\right) \\
& K_{3}^{T}(\lambda)=\psi_{n}\left(c_{0}\right) S\left(c_{0}\right) \xi\left(c_{0}\right)\left(\psi\left(c_{0}\right)-\psi(s)\right)
\end{aligned}
$$

First we have:

Lemma 3 The map $\zeta: x \longmapsto y=\xi(s(\lambda(x))) x$ is an homeomorphism from $\left\{x: \lambda(x) \geq \lambda_{1}\right\}$ to $\left\{y: k\left(\lambda_{1}\right) y^{T} D\left(\lambda_{1}\right) y \geq 1\right\}$. Moreover, the function $\tilde{\lambda}$ defined by

$$
\tilde{\lambda}(y)=\lambda\left(\zeta^{-1}(y)\right)=\lambda(x)
$$

is homogeneous of degree 1 with respect to the family of dilations defined by $\delta_{\alpha} y=\left(\alpha^{r_{1}} y_{1}\right.$, $\left.\ldots, \alpha^{r_{n}} y_{n}\right)$, in the sense that $\tilde{\lambda}\left(\delta_{\alpha} y\right)=\alpha \tilde{\lambda}(y)$ for any $y$ and $\alpha$ such that $y$ and $\delta_{\alpha} y$ belong to $\left\{y: k\left(\lambda_{1}\right) y^{T} D\left(\lambda_{1}\right) y \geq 1\right\}$. 
(Proof in Appendix)

In view of $(68),(74),(75)$, and Lemma 3 , we can rewrite $u(x)$ as

$$
u(x)=K^{T}(\lambda(x)) x=\sum_{i=1}^{3} K_{i}^{T}(\lambda(x)) y=\sum_{i=1}^{3} K_{i}^{T}(\tilde{\lambda}(y)) y
$$

Then we have:

Lemma 4 For any $i=1, \ldots, 3$, each component $K_{i, j}$ of the vector $K_{i}$ is a sum of functions of $\lambda$ homogeneous of degree not larger than $-r_{j}$.

(Proof in Appendix)

This lemma concludes the proof of Proposition 3. Indeed, in view of (77) and Lemma 3, $u$ as function of $y$ is a sum of functions homogeneous of non-positive degree with respect to the family of dilations $\left(\delta_{\alpha}\right)$. Since any continuous function that is homogeneous of nonpositive degree with respect to a family of dilations is bounded on any closed set that does not contain the origin, $u$ is therefore bounded.

\section{6 Ân illustrative example}

In this section, we illustrate our design method on the following system in $\mathbb{R}^{4}$ :

$$
\left\{\begin{array}{l}
\dot{x}_{1}=x_{2} \\
\dot{x}_{2}=x_{3} \\
\dot{x}_{3}=x_{4} \\
\dot{x}_{4}=-x_{3}+u .
\end{array}\right.
$$

for which we assume a magnitude limitation $|u| \leq 1$. Using (68), (69), and (28), the controller is given by $u(x)=u(\lambda(x), x)$ with

$$
u(\lambda, x)=-s_{1} s_{3}(\lambda) x_{1}-\left(s_{1}+s_{2}\right) s_{4}(\lambda) x_{2}-\left(s_{1}+s_{2}+s_{3}\right)(\lambda) x_{3}-s_{4}(\lambda) x_{4}+x_{3},
$$

with

$$
\lambda(x)= \begin{cases}1 & \text { if } V(1, x) \leq 1 \\ \text { the solution of } V(\lambda, x)=1 & \text { otherwise }\end{cases}
$$

and $V(\lambda, x)$ defined, in view of $(32),(27)$, and (29) by

$V(\lambda, x)=k(\lambda)\left[\left(s_{1} s_{2} s_{3}\right)(\lambda) x_{1}^{2}+\left(s_{2} s_{3}\right)(\lambda) x_{2}^{2}+s_{3}(\lambda)\left(x_{3}+s_{1}(\lambda) x_{1}\right)^{2}+\left(x_{4}+\left(s_{1}+s_{2}\right)(\lambda) x_{2}\right)^{2}\right]$.

The various parameters which define the functions $k$ and $s_{i}(i=1, \ldots, 4)$ have been chosen as follows:

$\eta=20$. This value has been obtained by simulation in order to satisfy the magnitude constraint $|u(x)| \leq 1$. Although we cannot guarantee that this constraint is satisfied with this value of $\eta$, this has always been the case for the simulations that we have done. 
$\lambda_{1}=2$.

$c_{1}=(1 / 5,4 / 5,5,4)^{T}$. This choice has been made in order to set all the eigenvalues equal to -1 in the domain where $u$ is linear (i.e., in $\{x: V(1, x) \leq 1\}$ ). Again, see [11] for instance for the algorithm to compute the components of $c_{1}$.

the vectors $\tau_{1}$ and $\tau_{2}$ have been defined in accordance with (38)-(39). In particular $\tau_{2}=(2,1,0,1 / 2)^{T}$, and in view of the definition of the other parameters, $\tau_{1}=$ $(0,0,(\ln 5) /(2 \ln 2), 1 / 2)^{T}$.

$c_{2}=(1 / 5,4 / 5,1,2)^{T}$. Since $m=2, c_{2,1}$ has to be chosen to ensure (43). A simple computation shows that this inequality is satisfied for any $c_{2,1}>0$ (because, for $i=1$, $C^{i+1} G^{i+1}$ is diagonal).

Finally, $\beta=\lambda_{2}=1$. The value $\lambda_{2}=1$ has first been obtained by following the procedure exposed at the end of Section 5.2. The value $\beta=1$ has also been obtained by following this procedure. Note that $\lambda_{2}=1, c_{2,1}=c_{1,1}$, and $c_{2,2}=c_{1,2}$ imply that $\tau_{1,1}=\tau_{1,2}=0$. This in turn implies that the matrix $\lambda \xi^{\prime} \psi(\lambda)$ in $(50)$ is zero (because $\xi(s)$ only depends on $s_{1}$ and $s_{2}$, which are constant if $\left.\tau_{1,1}=\tau_{1,2}=0\right)$. This accounts for the fact that finding $\beta$ is very easy in this case.

The following simulation result, with initial conditions $x(0)=(10,2,4,-4)^{T}$, illustrates the behavior of the controlled system (78)-(79). The implicit equation $V(\lambda, x)=1$ has been solved online by bisection.
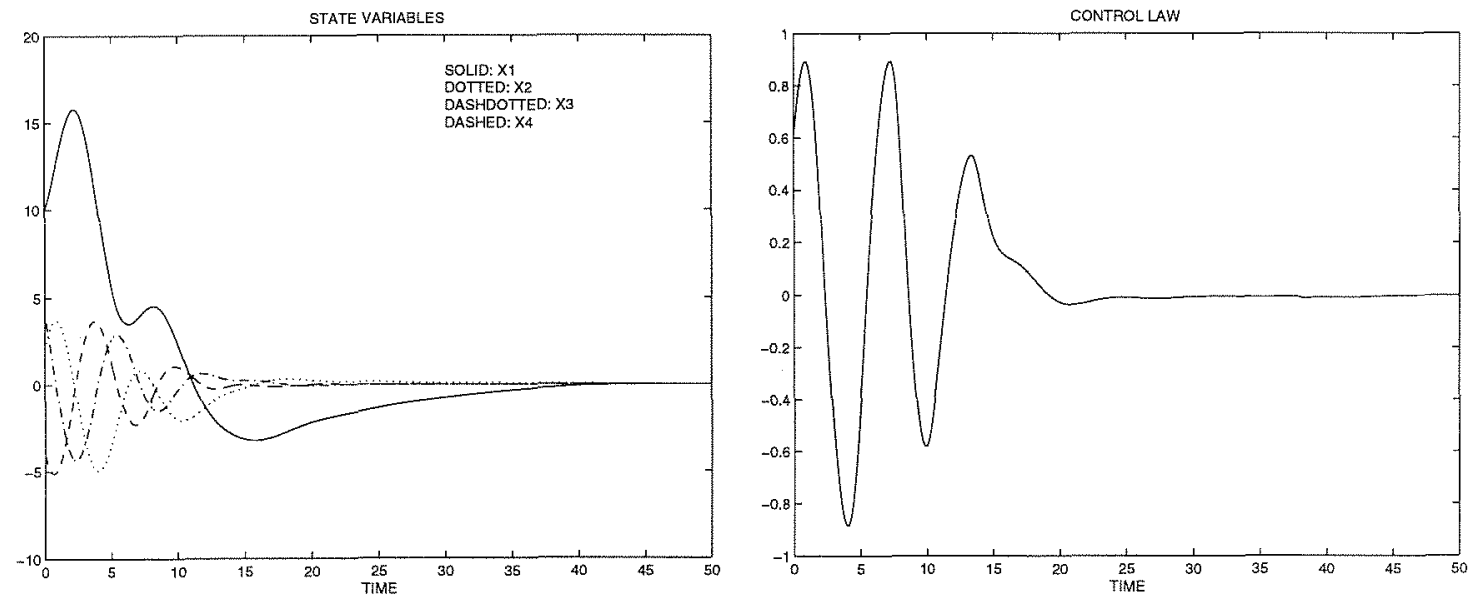


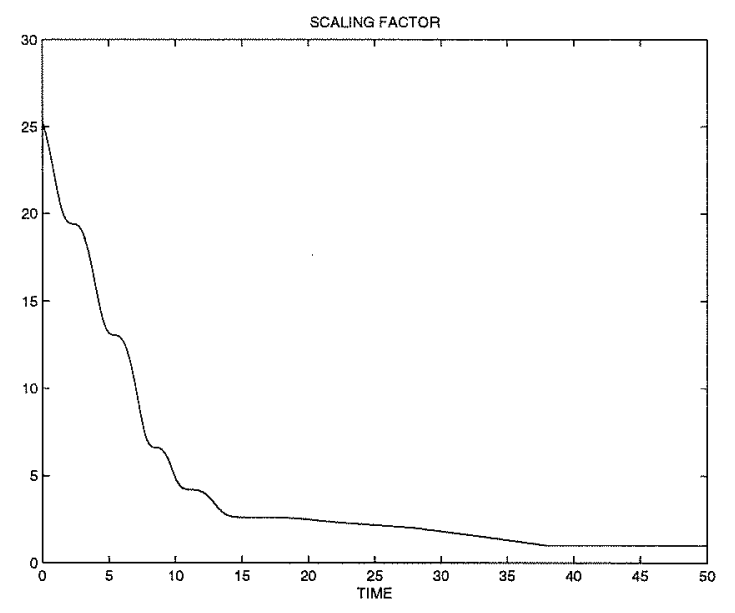

\section{Conclusion}

By properly rescaling a one-parameter family of linear control laws, we have obtained a constructive method for the design of bounded stabilizing feedbacks for single-input nullcontrollable linear systems. As shown by Theorem 1, this method is an application of a very general rescaling approach, some particular cases of which had been previously explored in [9] and [12] for the stabilization of homogeneous systems. The general form of Theorem 1 suggests many possible applications, including robust stabilization (i.e. with respect to unmodelled dynamics) or changing convergence rates. However, many issues need to be addressed in order to make the application of this method easier. Among them, the following are currently under investigation.

The "transversality condition" (Assumption 3) is in general difficult to satisfy. Obtaining weaker conditions, or simple ways to satisfy it, is an important open question.

For the problem of stabilization with magnitude limitations, it is important to relate the bound on the controller to the control parameters. This objective might require a different choice of the functions $s_{i}$. This might also require to better differentiate conditions on the $s_{i}$ 's that ensure stability, from conditions that ensure boundedness of the controller.

Again for this problem of stabilization with magnitude limitations, extension of the present result to multi-input linear systems, and to some classes of nonlinear systems needs to be considered.

Finally, extension of this result to magnitude and rate limitations should also be considered.

\section{Appendix}

\section{Proof of Theorem 1}

The proof of i) is essentially the same as the proof of $[9$, Th. 4]. We only give it for completeness.

The existence of a solution of (10) is a direct consequence of Assumption 1 and of the continuity of $V$. Let us show the uniqueness. Assume the existence, for some $(x, t) \in E_{1}$, 
of $\lambda_{1}$ and $\lambda_{2}\left(\lambda_{1}<\lambda_{2}\right)$ such that $V\left(\lambda_{i}, x, t\right)=1(i=1,2)$. Since $V$ is piecewise $\mathcal{C}^{1}$ and everywhere left and right differentiable with respect to $\lambda$, Assumption 3 implies that for some $\epsilon>0$,

$$
\begin{array}{ll}
V(\lambda, x, t)<1 & \forall \lambda \in\left(\lambda_{1}, \lambda_{1}+\epsilon\right) \\
V(\lambda, x, t)>1 & \forall \lambda \in\left(\lambda_{2}-\epsilon, \lambda_{2}\right)
\end{array}
$$

Since $V$ is continuous, there exists $\left.\lambda_{3} \in\right] \lambda_{1}, \lambda_{2}$ [ such that $V\left(\lambda_{3}, x, t\right)=1$. Iterating this argument, we conclude that between any two values of $\lambda$ for which $V(\lambda, x, t)=1, V(\lambda, x, t)=1$ for an infinite number of $\lambda$. By compactness of $\left[\lambda_{1}, \lambda_{2}\right]$ we deduce the existence of $\bar{\lambda}$ such that $V(\bar{\lambda}, x, t)=1$ and such that for any $\epsilon>0 V(\lambda, x, t)=1$ has an infinite number of solutions $\lambda \in[\bar{\lambda}-\epsilon, \bar{\lambda}+\epsilon]$. This is clearly in contradiction with Assumption 3.

We now prove ii). First, the $T$-periodicity of $\lambda$ is a direct consequence of the periodicity of $V$ and of the uniqueness of the solution of $(10)$. From now on, since the time-varying functions $u, V$, and $\lambda$, are $T$-periodic in the $t$ variable, we identify the time-space $\mathbb{R}$ with the compact set $\mathbb{R} / \mathbb{Z}=S^{1}$.

Before proving the continuity properties of $\lambda$, we first show that this function is radially unbounded with respect to $x$. In fact, for any $\lambda_{1}>\lambda_{0}$, the set $\left\{(x, t): V\left(\lambda_{1}, x, t\right) \leq 1\right\}$ is bounded with respect to $x$ uniformly in $t$ because $V$ is proper and $T$-periodic, and $\left\{(x, t): \lambda_{0}<\lambda(x, t) \leq \lambda_{1}\right\} \subset\left\{(x, t): V\left(\lambda_{1}, x, t\right) \leq 1\right\}$ since otherwise, there would exist $(x, t)$ such that $\lambda(x, t) \leq \lambda_{1}$ and $V\left(\lambda_{1}, x, t\right)>1$. This last inequality would imply the existence of $\lambda_{2}>\lambda_{1}$ such that $V\left(\lambda_{2}, x, t\right)=1$. This would be in contradiction with i).

Now we prove the continuity of $\lambda$ at $x=0$. Suppose on the contrary the existence of a sequence $\left(x_{n}, t_{n}\right) \longrightarrow(0, \bar{t})$ such that $\lambda\left(x_{n}, t_{n}\right) \nrightarrow \lambda(0, \bar{t})=\lambda_{0}$. Since the sequence $\left(x_{n}, t_{n}\right)$ is bounded and $\lambda$ is radially unbounded, the sequence $\lambda\left(x_{n}, t_{n}\right)$ is also bounded and there exists a subsequence $\left(x_{n_{p}}, t_{n_{p}}\right)_{p \in \mathbb{N}}$ such that $\lambda_{0}<\lambda\left(x_{n_{p}}, t_{n_{p}}\right) \longrightarrow \lambda_{1}>\lambda_{0}$. By continuity of $V$, we have therefore $1=V\left(\lambda\left(x_{n_{p}}, t_{n_{p}}\right), x_{n_{p}}, t_{n_{p}}\right) \longrightarrow V\left(\lambda_{1}, 0, \bar{t}\right)=0$. This is clearly a contradiction.

There only remains to prove that $\lambda$ is Lipschitz continuous on $\mathbb{R}^{n} \times S^{1}$ (resp. on $\left.\left(\mathbb{R}^{n} \backslash\{0\}\right) \times S^{1}\right)$ if $\lambda_{0} \in \Lambda$ (resp. if $\lambda_{0} \notin \Lambda$ ). First let us observe that when $\lambda_{0}$ is in $\Lambda$, we can extend the definition of $V$ to $\mathbb{R} \times \mathbb{R}^{n} \times \mathbb{R}$ by letting for instance for $\lambda \leq \lambda_{0}$

$$
V(\lambda, x, t)=\lambda_{0}-\lambda+V\left(\lambda_{0}, x, t\right) .
$$

Then, consider a point $(x, t)$ such that

$$
V(\lambda(x, t), x, t)=1
$$

From our assumptions, there exist $\eta>0$ and $k_{1}, k_{2}$, and $k_{3}$, such that for all $\left(\mu_{1}, y_{1}, s_{1}\right)$ and $\left(\mu_{2}, y_{2}, s_{2}\right)$ in $B((\lambda(x, t), x, t), \eta)$ we have, if $\mu_{1} \geq \mu_{2}$,

$$
\begin{aligned}
-k_{3}\left[\left|y_{1}-y_{2}\right|+\left|s_{1}-s_{2}\right|\right]-k_{1}\left|\mu_{1}-\mu_{2}\right| & \leq V\left(\mu_{1}, y_{1}, s_{1}\right)-V\left(\mu_{2}, y_{2}, s_{2}\right) \\
& \leq k_{3}\left[\left|y_{1}-y_{2}\right|+\left|s_{1}-s_{2}\right|\right]-k_{2}\left|\mu_{1}-\mu_{2}\right| .
\end{aligned}
$$

This implies that the function

$$
T(\mu, y, s)=\mu+\frac{1}{2 k_{1}}[V(\mu, y, s)-1]
$$


is a contraction in $\mu$. Therefore, for all $(y, s)$ in some neighborhood $\mathcal{N}$ of $(x, t)$, there exists a. unique $\mu(y, s)$ satisfying

$$
V(\mu, y, s)=1
$$

and moreover, from $(82)$, for all $\left(y_{1}, s_{1}\right)$ and $\left(y_{2}, s_{2}\right)$ in $\mathcal{N}$,

$$
\left|\mu\left(y_{1}, s_{1}\right)-\mu\left(y_{2}, s_{2}\right)\right| \leq \frac{k_{3}}{k_{2}}\left[\left|y_{1}-y_{2}\right|+\left|s_{1}-s_{2}\right|\right] .
$$

Then, for all $(y, s)$ in $\mathcal{N}$, by uniqueness of $\lambda(y, s)$ we have either

$$
\mu(y, s)<\lambda_{0}=\lambda(y, s) \leq \lambda(x, t)=\mu(x, t),
$$

or

$$
\mu(y, s)=\lambda(y, s) .
$$

This allows us to conclude that $\lambda$ is Lipschitz continuous on $\mathbb{R}^{n} \times \mathbb{R}$ when $\lambda_{0} \in \Lambda$ and on $\left(\mathbb{R}^{n} \backslash\{0\}\right) \times \mathbb{R}$ when $\lambda_{0} \notin \Lambda$.

In order to prove iii), we first show that the function $\lambda$ is non-increasing along the trajectories of the controlled system (8)-(12). Consider such a trajectory $(x(),$.$) and some$ time $t$ for which $V$ is differentiable ${ }^{2}$, at $(\lambda(x(t), t), x(t), t)$. Taking the derivative of (10) along this trajectory at $t$ yields:

$$
\frac{\partial V}{\partial \lambda} \dot{\lambda}=-\left[\frac{\partial V}{\partial x} \dot{x}+\frac{\partial V}{\partial t}\right]
$$

with $\dot{\lambda}$ the derivative of $\lambda$ along the trajectory. Assumption $A$ implies that the righthand side of $(83)$ is non-negative. If $(x(t), t) \in E_{1}^{o}=E_{1}$, Assumption 3 implies that the term $\frac{\partial V}{\partial \lambda}$ in the left-hand side is strictly negative. This implies that $\dot{\lambda}$ is non-positive. If $(x(t), t) \in E_{0}$ then $x(t)$ remains in this set ever-after because $\lambda$ is non-increasing on $E_{1}$ and because $\lambda(x(t), t)>\lambda_{0}$ for any $(x(t), t) \in E_{1}$. As a consequence, $\lambda=\lambda_{0}$ is also non-increasing on $E_{0}$.

Since $\lambda$ is non-increasing and proper with respect to $x$, the stability is guaranteed. Let us now consider any trajectory $(x(),$.$) of the system and show that x(t) \longmapsto 0$ as $t \longmapsto+\infty$. Since $\lambda$ is non-increasing along the trajectories of the system and since it is lower bounded by $\lambda_{0} \geq 0, \lambda$ converges to some value $\bar{\lambda}$.

First, suppose that $\bar{\lambda} \in \Lambda$. By application of Kurzweil's theorem [4], there exists a Lyapunov function $W \in \mathcal{C}^{1}\left(\mathbb{R}^{n}, \mathbb{R} ; \mathbb{R}\right), T$-periodic with respect to $t$, and such that

$$
\frac{\partial W}{\partial x}(x, t) f(x, u(\bar{\lambda}, x, t))+\frac{\partial W}{\partial t} \leq-U(x)
$$

with $U \mathcal{C}^{0}$, definite positive, and zero at the origin. Therefore,

$$
\begin{aligned}
\frac{\partial W}{\partial x} f(x, u(\lambda(x, t), x, t))+\frac{\partial W}{\partial t}= & \frac{\partial W}{\partial x} f(x, u(\bar{\lambda}, x, t))+\frac{\partial W}{\partial t} \\
& +\frac{\partial W}{\partial x}(f(x, u(\lambda(x, t), x, t))-f(x, u(\bar{\lambda}, x, t))) \\
& \leq-U(x)+g_{1}(x, t) g_{2}(\lambda(x, t)-\bar{\lambda}) \\
& \leq-U(x)+K \epsilon(t)
\end{aligned}
$$

\footnotetext{
${ }^{2}$ Note that it is sufficient to show that $\lambda$ is non-increasing for such values of $t$. Indeed, since $\lambda$ is continuous and $V$ is piecewise $\mathcal{C}^{1}$ w.r.t. $\lambda$, the map $t \longmapsto \lambda(x(t), t)$ cannot be locally increasing around a value $t_{0}$ without beeing increasing around some $t$ such that $V$ is differentiable at $(\lambda(x(t), t), x(t), t)$.
} 
where $g_{1}$ and $g_{2}$ are continuous functions with

$$
g_{2}(0)=0
$$

-which follows from the continuity of $f$ and $u-, g_{1}$ is $T$-periodic, $K$ is a constant, and $\epsilon$ is a function which tends to zero as $t$ tends to $+\infty$. The first inequality in (85) is obtained by application of the mean value theorem, using the fact that $f$ is $\mathcal{C}^{1}$, and $u$ is $\mathcal{C}^{0}$, periodic with respect to $t$, piecewise $\mathcal{C}^{1}$ and everywhere left and right differentiable with respect to $\lambda$ (Assumption $C$ ). The second inequality comes from the fact that the trajectory is bounded and $\lambda(x(t), t)$ tends to $\bar{\lambda}$ as $t$ tends to $\infty$. It follows from (85) that $W$ tends to zero and thus that $x(t)$ also tends to zero.

Finally, if $\bar{\lambda} \notin \Lambda$, then $\bar{\lambda}=\lambda_{0} \notin \Lambda$. If $x(t)$ does not converge to the origin then, since $x($.$) is bounded, there exists a subsequence \left(x\left(t_{n}\right), t_{n}\right)$ which tends to $(\bar{x}, \bar{t})$ with $\bar{x} \neq 0$. By continuity of $\lambda$, we have $\lambda_{0}=\bar{\lambda}=\lim _{n} \lambda\left(x\left(t_{n}\right), t_{n}\right)=\lambda(\bar{x}, \bar{t})$. Since $\lambda_{0} \notin \Lambda$, Assumption 2 implies that $E_{0}=\{0\} \times \mathbb{R}$. Since $\bar{x} \neq 0$, this implies that $(\bar{x}, \bar{t}) \in E_{1}$. By definition of $E_{1}$, $\lambda$ can not be equal to $\lambda_{0}$ on this set, and we obtain a contradiction.

\section{Proofs of Lemmas 2, 3, and 4}

The proofs are based on the following.

Claim 1: Let $i \in\{1, \ldots, n\}, j \in I_{i}$, and $\lambda \in\left[\lambda_{1},+\infty\right)$. Then,

a) If $i \in\{1, \ldots, m\}, \psi_{i, j}(s)$ is a sum of monomials in $\lambda$ of degree $-(i-j) \tau_{2, m-1}$.

b) $\psi_{i, j}(s)-\psi_{i, j}\left(c_{0}\right)$ is a sum of monomials in $\lambda$ of degree

$$
\begin{cases}-(i-j) \tau_{2, m-1} & \text { if } i \in\{1, \ldots, m\}, \\ \text { not larger than }-r_{j} & \text { otherwise. }\end{cases}
$$

c) $\xi_{i, j}(s)$ is a sum of monomials in $\lambda$ of degree

$$
\begin{cases}-(i-j) \tau_{2, m-1} & \text { if } i \in\{1, \ldots, m\} \\ \text { not larger than }-r_{j} & \text { if } i>m \text { and } j \leq m .\end{cases}
$$

Proof: Let us prove a). In view of (28), one easily verifies by induction the two following properties:

1. each $\psi_{i, j}(s)$ is a sum of monomials in the $s_{i}$ of degree $\frac{i-j}{2}$.

2. these monomials contain no $s_{k}: k>i-2$.

Since, for $k \leq m-1$, each $s_{k}$ is a monomial of degree $-2 \tau_{2, m-1}$ in $\lambda$ (see (37) - (39)), the two properties above imply that for $i \leq m, \psi_{i, j}(s)$ is a sum of monomials in $\lambda$ of degree $\frac{i-j}{2}\left(-2 \tau_{2, m-1}\right)=-(i-j) \tau_{2, m-1}$.

We prove b). We first assume that $i \leq m$. Using Properties 1 and 2 above, we deduce that for $j<i, \psi_{i, j}\left(c_{0}\right)=0$ because $c_{0, k}=0$ for $k \leq m$. In view of a), this implies that the claim is satisfied for $j<i$. For $i=j$, b) is also satisfied since $\psi_{i, i}($.$) is constant (\equiv 1)$. 
For $i \in\{m+1, \ldots, n\}$, we proceed by induction on $i$. If $m \leq 1$ then $m+1 \leq 2$ and it is direct to verify that $\psi_{m+1, j}(s)-\psi_{m+1, j}\left(c_{0}\right)=0$ so that the claim holds. If $m \geq 2$, we have from (28)

$$
\psi_{m+1, j}(s)-\psi_{m+1, j}\left(c_{0}\right)= \begin{cases}-s_{1} \psi_{m, 2}(s)+c_{0,1} \psi_{m, 2}\left(c_{0}\right) & (j=1), \\ -s_{j} \psi_{m, j+1}(s)+c_{0, j} \psi_{m, j+1}\left(c_{0}\right) & \\ +\psi_{m, j-1}(s)-\psi_{m, j-1}\left(c_{0}\right) & \left(1<j<m+1, j \in I_{i}\right), \\ 0 & (j=m+1) .\end{cases}
$$

In view of the claim for $i \in\{1, \ldots, m\}$,

$$
\operatorname{deg}\left(\psi_{m, j-1}(s)-\psi_{m, j-1}\left(c_{0}\right)\right)=-(m-(j-1)) \tau_{2, m-1}=-(m+1-j) \tau_{2, m-1}=-r_{j}
$$

where the last equality comes from the fact that for $j \leq m-1$,

$$
r_{j}=\sum_{k=j}^{m-1} \tau_{2, k}+\sum_{k=m}^{n-1} \tau_{2, k}+1=\sum_{k=j}^{m-1} \tau_{2, m-1}+\tau_{2, m-1}=(m+1-j) \tau_{m-1} .
$$

Consider now the terms of the form $-s_{j} \psi_{m, j+1}(s)+c_{0, j} \psi_{m, j+1}\left(c_{0}\right)$ in (88). First, $c_{0, j}=0$ because $j \leq m$. Therefore,

$$
\begin{aligned}
\operatorname{deg}\left(-s_{j} \psi_{m, j+1}(s)+c_{0, j} \psi_{m, j+1}\left(c_{0}\right)\right) & =\operatorname{deg}\left(-s_{j} \psi_{m, j+1}(s)\right)=-2 \tau_{2, j}-(m-j-1) \tau_{2, m-1} \\
& =-(m-j+1) \tau_{2, m-1}=-r_{j}
\end{aligned}
$$

where the last equality comes from (90).

We now assume that the claim holds up to $i \geq m+1$ and prove it for $i+1$. (88), with $i$ instead of $m$, is still satisfied. Using the induction hypothesis, we have

$$
\operatorname{deg}\left(\psi_{i_{i} j-1}(s)-\psi_{i, j-1}\left(c_{0}\right)\right) \leq-r_{j-1} \leq-r_{j}
$$

Then, if $c_{0, j}=0$,

$$
\operatorname{deg}\left(-s_{j} \psi_{i, j+1}(s)+c_{0, j} \psi_{i, j+1}\left(c_{0}\right)\right)=\operatorname{deg}\left(-s_{j}\left(\psi_{i, j+1}(s)-\psi_{i, j+1}\left(c_{0}\right)\right)-s_{j} \psi_{i, j+1}\left(c_{0}\right)\right) \text {. }
$$

By the induction assumption,

$$
\operatorname{deg}\left(-s_{j}\left(\psi_{i, j+1}(s)-\psi_{i, j+1}\left(c_{0}\right)\right)\right) \leq-2 \tau_{j}-r_{j+1}=-r_{j}-\tau_{2, j}<-r_{j} .
$$

Consider now the term $s_{j} \psi_{i, j+1}\left(c_{0}\right)$ in the right-hand side of (93). If $j \leq m-1$ we claim that this term is zero. This comes from the fact that $c_{0, k}=0$ for any $\bar{k} \leq m$, and, from (28), each $\psi_{i, j}(s)$ (for $i>j$ ) contains at least one $s_{k}: k \leq j$. If $j>m-1$, then $i>m-1$ and (39)-(41) imply that $2 \tau_{2, j}=r_{j}$ so that,

$$
\operatorname{deg}\left(s_{j} \psi_{i, j+1}\left(c_{0}\right)\right)=-2 \tau_{2, j}=-r_{j}
$$

Finally, if $c_{0, j} \neq 0,(37),(39)$, and (42) imply that $s_{j}=c_{0, j}$ and $r_{j}=r_{j+1}$ so that

$$
\operatorname{deg}\left(-s_{j} \psi_{i, j+1}(s)+c_{0, j} \psi_{i, j+1}\left(c_{0}\right)\right)=\operatorname{deg}\left(\psi_{i, j+1}(s)-\psi_{i, j+1}\left(c_{0}\right)\right) \leq-r_{j+1}=-r_{j} .
$$


The proof of $b$ ) is now complete.

There remains to prove $c$ ). First, remark that for $m=0$ there is nothing to prove. Therefore we assume $m>0$. For $i \leq m$, the proof is similar to the proof of a). In particular, $\xi_{i, j}(s)$ is also a sum of monomials in the $s_{i}$ of degree $\frac{i-j}{2}$, and each of these monomials contains no $s_{k}: k>i-2$. For $i \in\{m+1, \ldots, n\}$, we proceed by induction. If $m=1$ then the claim holds for $i=m+1$ since in this case, $\left\{(i, j): i=m+1, j \leq m, j \in I_{i}\right\}=\{\emptyset\}$. If $m>1$, we have from (29),

$$
\xi_{m+1, j}(s)= \begin{cases}s_{m-1} \xi_{m-1,1} & (j=1) \\ \xi_{m, j-1}(s)+s_{m-1} \xi_{m-1, j} & (j>1) .\end{cases}
$$

In view of the claim for $i \in\{1, \ldots, m\}$, the terms $s_{m-1} \xi_{m-1, j}$ are of degree $-2 \tau_{m-1}-(m-$ $1-j) \tau_{2, m-1}=-(m+1-j) \tau_{2, m-1}=-r_{j}$ where the last equality comes from (90) and from the fact that $j \leq m-1$ since $j \in I_{m+1}$ and $j \leq m$. The terms $\xi_{m, j-1}(s)$ are also of degree $-(m-(j-1)) \tau_{2, m-1}=-(m+1-j) \tau_{2, m-1}=-r_{j}$.

We now assume that the claim is true up to $i \geq m+1$ and prove it for $i+1$. (97) is still satisfied with $i$ instead of $m$. Using the induction assumption, the terms $s_{i-1} \xi_{i-1, j}$ are of degree:

$$
\begin{array}{ll}
-2 \tau_{2, m}-(m-j) \tau_{2, m-1}=-(m+1-j) \tau_{2, m-1} & (i=m+1), \\
\text { not larger than }-r_{j} & (i>m+1) .
\end{array}
$$

If $j<m$ then, in view of $(90),-(m+1-j) \tau_{2, m-1}=-r_{j}$. If $j=m,-(m+1-j) \tau_{2, m-1}=$ $-\tau_{2, m-1}=-r_{m}$ where the last equality comes from (39) and (41). Finally, the terms $\xi_{i, j-1}(s)$ are of degree not larger than $-r_{j-1}$ and therefore, not larger than $-r_{j}$ since $r_{j-1} \geq r_{j}$.

Proof of Lemma 2: In view of (55),

$$
\tilde{n}_{i, j}=\left(\prod_{k=i}^{n-1} c_{2, k}\right) \tilde{\lambda}^{r_{j}-r_{i}}\left(-R_{i, j}+\tilde{\lambda}\left(\tilde{\xi}^{o} \tilde{\psi}\right)_{i, j}\right)
$$

with

$$
\left(\tilde{\xi}^{\circ} \tilde{\psi}\right)_{i, j}=\sum_{i>k \geq j} \frac{\partial}{\partial \tilde{\lambda}} \xi_{i, k}(\tilde{s}(\tilde{\lambda})) \psi_{k, j}(\tilde{s}(\tilde{\lambda})) .
$$

The last equality comes from (56), and from the fact that $\xi_{i, j}=\psi_{i, j}=0$ if $j \geq i$, and $\xi_{i, i} \equiv 1$.

First, $\left(\tilde{\xi}^{\circ} \tilde{\psi}\right)_{i, j}$ is zero if $j \notin I_{i}$ since in this case, whether $j>i$ and the sum in $(100)$ is taken on the empty set, or $i-j$ is odd in which case, for any $k, i-k$ or $k-j$ is also odd and therefore, each product in the last sum of (100) is zero. From (41), $R=\operatorname{Diag}\left(r_{i}\right)$ so that Property i) of Lemma 2 readily follows.

Property ii) is also a direct consequence since for $i=j$, the last sum in (100) is taken on the empty set.

In view of (99),

$$
\left(\prod_{k=i}^{n-1} \frac{1}{c_{2, k}}\right) \tilde{n}_{i, j}=\tilde{\lambda}^{r_{j}-r_{i}}\left(-R_{i, j}+\tilde{\lambda}\left(\tilde{\xi}^{o} \tilde{\psi}\right)_{i, j}\right)
$$


So, in order to prove Property iii), all we need to do is to show that $\tilde{\lambda}\left(\tilde{\xi}^{\circ} \tilde{\psi}\right)_{i, j}$ does not depend on the $c_{2, k}: k \geq i-1$. This is a direct consequence of (100) and of the fact (mentioned in the proof of Claim 1) that both $\psi_{i, j}(s)$ and $\xi_{i, j}(s)$ do not depend on the $s_{k}: k \geq i-1$.

Since $R$ is diagonal, the proof of iv) consists in showing that $\tilde{\lambda}^{r_{j}-r_{i}} \tilde{\lambda}\left(\tilde{\xi}^{o} \tilde{\psi}\right)_{i, j}$ is independent of $\tilde{\lambda}$. From Claim 1 , each $\xi_{i, k}(s)$ is a sum of monomials in $\lambda$ of degree $-(i-k) \tau_{2, m-1}$ (because $i \leq m)$. This implies, using (44), that $\xi_{i, k}(\tilde{s})$ is also a sum of monomials in $\tilde{\lambda}$ of degree $-(i-j) \tau_{2, m-1}$. Similarly, using Claim 1 again, for $k<i \leq m, \psi_{k, j}(\tilde{s})$ is a sum of monomials in $\tilde{\lambda}$ of degree $-(k-j) \tau_{2, m-1}$. This implies, in view of $(100)$, that $\tilde{\lambda}\left(\tilde{\xi}^{o} \tilde{\psi}\right)_{i, j}$ is a sum of monomials in $\tilde{\lambda}$ of degree

$$
-(i-j) \tau_{2, m-1}=-\left(r_{j}-r_{i}\right)
$$

where the last equality comes from (39), (41), and the fact that both $i$ and $j$ are not larger than $m$.

Finally we prove v). In view of (99), it is clearly sufficient to show that for any $i>m$ and $j \in I_{i}, \tilde{\lambda}\left(\tilde{\xi}^{\circ} \tilde{\psi}\right)_{i, j}$ is a sum of monomials in $\tilde{\lambda}$ of degree not larger than $-r_{j}$. Using the fact that $\xi$ is the inverse of $\psi$, we have

$$
\tilde{\lambda}\left(\tilde{\xi}^{o} \tilde{\psi}\right)_{i, j}=-\tilde{\lambda}\left(\tilde{\xi} \tilde{\psi}^{o}\right)_{i, j}=-\tilde{\lambda} \sum_{m \geq k>j} \tilde{\xi}_{i, k} \tilde{\psi}_{k, j}^{o}-\tilde{\lambda} \sum_{k>m} \tilde{\xi}_{i, k} \tilde{\psi}_{k, j}^{o}
$$

We consider the first sum in the right hand side of (102). Since $i>m$ and $k \leq m$, Claim 1 implies that each $\tilde{\xi}_{i, k}$ is a sum of monomials in $\tilde{\lambda}$ of degree not larger than $-r_{k}$, and each $\tilde{\psi}_{k, j}^{o}$ is a sum of monomials of degree $-(k-j) \tau_{2, m-1}-1$ (the term -1 coming from the derivation). As a consequence, each term $\tilde{\lambda} \tilde{\xi}_{i, k} \tilde{\psi}_{k, j}^{o}$ in the first sum of (102) is a sum of monomials of degree not larger than $-r_{k}-(k-j) \tau_{2, m-1}=-r_{j}$ where the last equality comes from the fact that, since $m \geq k>j, r_{j}=r_{k}+(k-j) \tau_{2, m-1}$.

Finally, we consider the second sum in the right hand side of (102). Since $k>m$, Claim 1 implies that $\tilde{\lambda} \tilde{\psi}_{k, j}^{o}$ is a sum of monomials of degree not larger than $-r_{j}$. Moreover, $\tilde{\xi}_{i, k}$ is a sum of monomials of non-positive degree since each $s_{i}$ is a monomial in $\lambda$ of non-positive degree and since $\tilde{\xi}_{i, k}$ is a polynomial in the $s_{i}$ 's. Therefore, each term in the second sum of (102) is also a sum of monomials in $\tilde{\lambda}$ of degree not larger than $-r_{j}$. This concludes the proof of $\mathrm{v})$.

Proof of Lemma 3: We first prove that $\zeta$ is an homeomorphism.

To begin with, for any $y \in\left\{y: k\left(\lambda_{1}\right) y^{T} D\left(\lambda_{1}\right) y \geq 1\right\}$, there exists $\tilde{\lambda}(y) \geq \lambda_{1}$ such that $k(\tilde{\lambda}(y)) y^{T} D(\tilde{\lambda}(y)) y=1$ : this comes from the fact that, in view of (36) and (27), $k(\lambda) y^{T} D(\lambda) y$ tends to zero as $\lambda$ tends to $+\infty$. Let $x=\psi(\tilde{\lambda}(y)) y$ so that $y=\xi(\tilde{\lambda}(y)) x$. Then,

$$
k(\tilde{\lambda}(y)) x^{T} \xi^{T}(\tilde{\lambda}(y)) D(\tilde{\lambda}(y)) \xi(\tilde{\lambda}(y)) x=1 .
$$

In view of the definition of the function $\lambda$, this implies that $\tilde{\lambda}(y)=\lambda(x)$. Therefore $\zeta(x)=y$ and $\zeta$ is onto.

We show that $\zeta$ is injective. Suppose that for some $x_{1} \neq x_{2}, \xi\left(\lambda\left(x_{1}\right)\right) x_{1}=\xi\left(\lambda\left(x_{2}\right)\right) x_{2}$. First, $\lambda\left(x_{1}\right) \neq \lambda\left(x_{2}\right)$ since otherwise, $x_{1}=x_{2}$ because $\xi(\lambda)$ is bijective for any $\lambda$. Therefore, there exists $\lambda\left(x_{1}\right) \neq \lambda\left(x_{2}\right)$ such that

$$
k\left(\lambda\left(x_{1}\right)\right) y^{T} D\left(\lambda\left(x_{1}\right)\right) y=k\left(\lambda\left(x_{2}\right)\right) y^{T} D\left(\lambda\left(x_{2}\right)\right) y .
$$


This is impossible because the map $\lambda \longmapsto k(\lambda) y^{T} D(\lambda) y$ is strictly decreasing for any $y \neq 0$.

Now we show that $\tilde{\lambda}$ is homogeneous of degree one with respect to the family of dilations defined by $\delta_{\alpha} y=\left(\alpha^{r_{1}} y_{1}, \ldots, \alpha^{r_{n}} y_{n}\right)$. Indeed, $\tilde{\lambda}$ is defined by

$$
k(\tilde{\lambda}) y^{T} D(\tilde{\lambda}) y=\eta \lambda_{1}^{2(1-p)} \lambda^{-2} y^{T} D(\tilde{\lambda}) y=1 .
$$

Using the definitions $(27),(37)$, and (41) of $D, s$, and $r_{i},(104)$ is equivalent to

$$
\sum_{i=1}^{n} \beta_{i}\left(\frac{y_{i}}{\tilde{\lambda}^{r_{i}}(y)}\right)^{2}=1
$$

with $\beta_{i}(i=1, \ldots, n)$ independent of $y$ and $\tilde{\lambda}$. Therefore, we also have

$$
\sum_{i=1}^{n} \beta_{i}\left(\frac{\alpha^{r_{i}} y_{i}}{\tilde{\lambda}^{r_{i}}\left(\delta_{\alpha} y\right)}\right)^{2}=1=\sum_{i=1}^{n} \beta_{i}\left(\frac{y_{i}}{\left(\alpha^{-1} \tilde{\lambda}\right)^{r_{i}}\left(\delta_{\alpha} y\right)}\right)^{2} .
$$

Since $\tilde{\lambda}(y)$ is uniquely defined, we deduce from (105) and (106) that

$$
\alpha^{-1} \tilde{\lambda}\left(\delta_{\alpha} y\right)=\tilde{\lambda}(y)
$$

which precisely means that $\tilde{\lambda}$ is homogeneous of degree 1 .

Proof of Lemma 4: We first consider $K_{1}$. In view of $(37),(39)$, and $(42), c_{0, j} \neq 0 \Longrightarrow$ $c_{0, j}=s_{j}(\lambda)\left(\lambda \in\left[\lambda_{1},+\infty\right)\right)$. Therefore,

$$
\left(S(s)-S\left(c_{0}\right)\right)_{i, j}= \begin{cases}-s_{j} & \left(i=j+1 \leq n \text { and } c_{0, j}=0\right) \\ -s_{n} & \left(i=j=n \text { and } c_{0, j}=0\right) \\ 0 & \text { otherwise }\end{cases}
$$

This implies that

$$
K_{1, j}= \begin{cases}-s_{j} \psi_{n, j+1}(s) & \left(j<n \text { and } c_{0, j}=0\right), \\ -s_{n} \psi_{n, n}(s)=-s_{n} & \left(j=n \text { and } c_{0, j}=0\right) \\ 0 & \text { otherwise }\end{cases}
$$

Let us first assume that $m=n$, i.e. $c_{0}=0$. In this case, using (108), (39), and (41),

$$
\begin{aligned}
& \operatorname{deg}\left(K_{1, n-1}\right)=\operatorname{deg}\left(s_{n-1}\right)=-2 \tau_{2, n-1}=-2=-r_{n-1}, \\
& \operatorname{deg}\left(K_{1, n}\right)=\operatorname{deg}\left(s_{n}\right)=-2 \tau_{2, n}=-1=-r_{n} .
\end{aligned}
$$

Using also the fact that $\psi\left(c_{0}\right)=\psi(0)=\operatorname{Id}(n)$, we obtain for $j<n-1$ and $c_{0, j}=0$

$$
\begin{aligned}
\operatorname{deg}\left(K_{1, j}\right) & =\operatorname{deg}\left(s_{j} \psi_{n, j+1}(s)\right)=\operatorname{deg}\left(s_{j}\left(\psi_{n, j+1}(s)-\psi_{n, j+1}\left(c_{0}\right)\right)\right) \\
& =\operatorname{deg}\left(s_{j}\right)-(n-(j+1)) \tau_{2, m-1}=-2 \tau_{2, m-1}-(n-(j+1)) \tau_{2, m-1} \\
& =-(n-j+1) \tau_{2, m-1}=-r_{j} .
\end{aligned}
$$

where we have used Claim 1 , and the fact that $m=n \Longrightarrow \tau_{2, j}=\tau_{2, m-1}=1$ for $j<n$. 
If $m<n$, we first remark that $\psi_{n, j}\left(c_{0}\right)=0$ for $j \leq m$ : this comes from the fact that $c_{0, k}=0$ for $k \leq m$ and that $\psi_{i, j}(s)$ (for any $i>j$ and any $s$ ) is a polynomial in $s$ which monomials contain at least one of the $s_{k}(k \leq j)$ (in view of (28), this readily follows by induction on $i$ ). As a consequence, for $j \leq m-2<n, \psi_{n, j+1}(s)=\psi_{n, j+1}(s)-\psi_{n, j+1}\left(c_{0}\right)$ so that, in view of Claim 1 ,

$$
\operatorname{deg}\left(K_{1, j}\right)=\operatorname{deg}\left(s_{j} \psi_{n, j+1}(s)\right) \leq-2 \tau_{2, j}-r_{j+1} \leq-\left(r_{j+1}+2 \tau_{2, j}\right) \leq-r_{j}
$$

where the last inequality comes from the fact that $r_{j}=r_{j+1}+\tau_{2, j}$. For $j>m-2$ and $c_{0, j}=0$,

$$
\operatorname{deg}\left(K_{i, j}\right) \leq \operatorname{deg}\left(s_{j}\right)=-2 \tau_{2, j}=-r_{j} .
$$

The first inequality above comes from the fact that each $\psi_{i, j}$ is a sum of polynomials functions in the $s_{i}$ 's, each of which is a polynomial of non-positive degree in $\lambda$. The last equality is a direct consequence of (39)-(41). The proof of the claim for $K_{1}$ is now complete.

Next, let us consider $K_{2}$. In view of (75) and (24),

$$
K_{2, j}= \begin{cases}\left(\psi_{n, 2}(s)-\psi_{n, 2}\left(c_{0}\right)\right) c_{0,1} & (j=1), \\ \left(\psi_{n, j-1}(s)-\psi_{n, j-1}\left(c_{0}\right)\right)+\left(\psi_{n, j+1}(s)-\psi_{n, j+1}\left(c_{0}\right)\right) c_{0, j} & (1<j<n), \\ \left(\psi_{n, n-1}(s)-\psi_{n, n-1}\left(c_{0}\right)\right)+\left(\psi_{n, n}(s)-\psi_{n, n}\left(c_{0}\right)\right) c_{0, j} & (j=n) .\end{cases}
$$

First, in view of Claim 1, each term of the form $\left(\psi_{n, j-1}(s)-\psi_{n, j-1}\left(c_{0}\right)\right)$ in (113) is of degree not larger than $-r_{j}$. This is a direct consequence of Claim 1 if $m<n$, and of Claim 1 and $(90)$ if $m=n$. Then, $\left(\psi_{n, n}(s)-\psi_{n, n}\left(c_{0}\right)\right) c_{0, j}=0$ since $\psi_{n, n}(s)=\psi_{n, n}\left(c_{0}\right)=1$. Finally, each term of the form $\left(\psi_{n, j+1}(s)-\psi_{n, j+1}\left(c_{0}\right)\right) c_{0, j}$ is zero if $c_{0, j}=0$ or, in view of Claim 1, homogeneous of degree not larger than $-r_{j+1}=-r_{j}$ since in view of (39)-(41), $c_{0, j} \neq 0 \Longrightarrow \tau_{2, j}=0 \Longrightarrow r_{j+1}=r_{j}$.

There remains to consider $K_{3}$. If $m=n$ then $\psi\left(c_{0}\right)=\xi\left(c_{0}\right)=I$ so that $K_{3}=$ $S_{n}\left(c_{0}\right)\left(\psi\left(c_{0}\right)-\psi(s)\right)=0$ since $c_{0}=0 \Longrightarrow S_{n}\left(c_{0}\right)=0$.

If $m \neq n$, we first show that

$$
\left(\psi_{n}\left(c_{0}\right) S\left(c_{0}\right) \xi\left(c_{0}\right)\right)_{k}=0 \quad \forall k \leq m
$$

Indeed, we have already mentioned that $\psi_{i, j}\left(c_{0}\right)=0$ for all $j \leq m(j \neq i)$. This implies that $\psi\left(c_{0}\right)$ has a block diagonal structure:

$$
\psi\left(c_{0}\right)=\left(\begin{array}{cc}
\operatorname{Id}(m) & 0 \\
0 & B
\end{array}\right) .
$$

As a consequence, $\xi\left(c_{0}\right)=\psi^{-1}\left(c_{0}\right)$ has the same block diagonal structure, i.e., $\xi_{i, j}\left(c_{0}\right)=0$ for all $j \leq m(j \neq i)$. This implies that, for $k \leq m$,

$$
\begin{aligned}
\left(\psi_{n}\left(c_{0}\right) S\left(c_{0}\right) \xi\left(c_{0}\right)\right)_{k} & =\sum_{j}\left(\psi_{n}\left(c_{0}\right) S\left(c_{0}\right)\right)_{j} \xi_{j, k}=\left(\psi_{n}\left(c_{0}\right) S\left(c_{0}\right)\right)_{k}=\sum_{q>k} \psi_{n, q}\left(c_{0}\right) S_{q, k}\left(c_{0}\right) \\
& =\psi_{n, k+1}\left(c_{0}\right) S_{k+1, k}\left(c_{0}\right)=\psi_{n, k+1}\left(c_{0}\right) c_{0, k}=0
\end{aligned}
$$

which proves (114). Since Claim 1 implies that $\operatorname{deg}\left(\psi\left(c_{0}\right)-\psi(s)\right)_{k, j} \leq-r_{j}$ for $k>m$, the Claim for $K_{3}$ readily follows. 


\section{References}

[1] C.-T. Chen, Linear system theory and design, Oxford University Press, 1984.

[2] H. Hermes, Nilpotent and high-order approximations of vector field systems, SIAM Review, Vol. 33, pp. 238-264, 1991.

[3] M. Kawski, Geometric homogeneity and stabilization, IFAC Symp. on Nonlinear Control System Design (NOLCOS), pp. 164-169, 1995.

[4] J. Kurzweil, On the inversion of Lyapunov's second theorem on stability of motion, Ann. Math. Soc., Transl. Ser. 2, 24, pp. 19-77, 1956.

[5] J.P. LaSalle, Stability Theory for Ordinary Differential Equations, Journal of Differential Equations, Vol. 4, pp. 57-65, 1968.

[6] T. Lauvdal, R.M. Murray, T.I. Fossen, Stabilization of integrator chains in the presence of magnitude and rate saturations, 36th IEEE Conf. on Decision and Control (to appear), 1997.

[7] S.G. Loo, A simplified proof of a transformation matrix relating the companion matrix and the Schwartz matrix, IEEE Trans, on Automatic Control, Vol. 13, pp. 309-310, June 1968.

[8] Z. Lin, Global control of linear systems with saturating actuators, 35th IEEE Conf. on Decision and Control, pp. 4357-4362, 1996.

[9] R.T. M'Closkey, R.M. Murray, Exponential stabilization of driftless nonlinear control systems using homogeneous feedback, IEEE Trans. on Automatic Control, Vol. 42, pp. 614-628, May 1997.

[10] A. Megretski, $L_{2}$ BIBO Output feedback stabilization with saturated control, $13 t h$ IFAC World Congress, Vol. D, pp. 435-440, 1996.

[11] P. Morin, C. Samson, Control of nonlinear chained systems. From the Routh-Hurwitz stability criterion to time-varying exponential stabilizers, 36th IEEE Conf. on Decision and Control (to appear), 1997.

[12] L. Praly, Generalized weighted homogeneity and state dependent time scale for linear controllable systems, 36th IEEE Conf. on Decision and Control (to appear), 1997.

[13] L. Rosier, General symmetries for ordinary differential equations. Application to stability and controllability, in Etudes de quelques problemes de stabilisation, Ph.D Dissertation, Ecole Normale Superieure de Cachan, France, 1993.

[14] I.G. Sarma, M.A. Pai, R. Viswanathan, On the transformation to Schwartz canonical form, IEEE Trans. on Automatic Control, Vol. 13, pp. 311-312, June 1968.

[15] H.J. Sussmann, E.D. Sontag, Y. Yang, A general result on the stabilization of linear systems using bounded controls, IEEE Trans. on Automatic Control, Vol. 39, pp. 2411-2425, 1994. 
[16] A.R. Teel, Global stabilization and restricted tracking for multiple integrators with bounded controls, Systems \& Control Letters, Vol. 18, pp. 165-171, 1992.

[17] A.R. Teel, Linear systems with input nonlinearities: global stabilization by scheduling a family of $\mathcal{H}_{\infty}$-type controllers, Int. J. of Robust and Nonlinear Control, Vol. 5, pp. $399-411,1995$. 